\title{
G

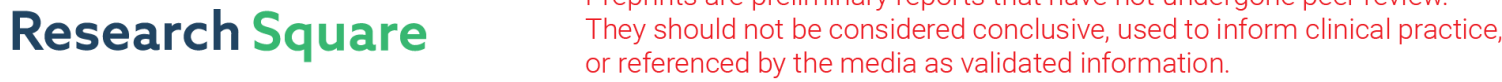 \\ Osteoblasts-derived Exosomes as Novel Communicators in Particle Induced Periprosthetic Osteolysis
}

\section{Wanderson de Souza}

Inmetro: Instituto Nacional de Metrologia Qualidade e Tecnologia https://orcid.org/0000-0002-96324928

\section{Sara G. Piperni}

Universidade do Grande Rio

\section{Carolina F. Ruivo}

I3S: Universidade do Porto Instituto de Investigacao e Inovacao em Saude

\section{Nuno Bastos}

I3S: Universidade do Porto Instituto de Investigacao e Inovacao em Saude

\section{Patrícia Cardoso}

I3S: Universidade do Porto Instituto de Investigacao e Inovacao em Saude

\section{Maria J. Oliveira}

I3S: Universidade do Porto Instituto de Investigacao e Inovacao em Saude

\section{Luís A. Rocha}

Universidade Estadual Paulista Julio de Mesquita Filho

José M. Granjeiro

Inmetro: Instituto Nacional de Metrologia Qualidade e Tecnologia

\section{Maria H. Fernandes}

Universidade do Porto Faculdade de Medicina Dentaria

\section{Sonia A. Melo}

I3S: Universidade do Porto Instituto de Investigacao e Inovacao em Saude

Ana R. Ribeiro ( $\sim$ ana.ribeiro@inl.int )

University Grande Rio https://orcid.org/0000-0003-1349-9595

\section{Research}

Keywords: Titanium dioxide, nanoparticles, exosomes, osteoblasts, macrophages, inflammation, proteomic biomarkers

Posted Date: July 27th, 2021

DOI: https://doi.org/10.21203/rs.3.rs-657724/v1 
License: (c) (i) This work is licensed under a Creative Commons Attribution 4.0 International License. Read Full License 


\section{Abstract}

\section{Background}

The inflammatory response to titanium implant-derived wear particles is considered as the hallmark of periprosthetic osteolysis, an event that cause pain, reduce patient motility, ultimately leading to the need of a revision surgery. Although macrophages are major cell players, other cell types such as bone cells can indirectly contribute to periprosthetic osteolysis, however the mechanisms are not fully understood. Exosomes (Exos) has been related with several bone pathologies, with growing body of literature recognizing them as actively shuttle molecules through the body, with their cargo being completely dependent of external stimuli (e.g. chemicals and metals ions and particles). Till the moment, the role of wear debris on osteoblasts exosomes biogenesis is absent and the possible contribution of Exos to osteoimmune communication and periprosthetic osteolysis is still in its infancy. Taking that in consideration, in this work we investigate the effect of wear debris on Exo biogenesis, where two bone cell models were exposed to titanium dioxide nanoparticles $\left(\mathrm{TiO}_{2} \mathrm{NPs}\right)$ similar in size and composition to wear debris associated with prosthetic implants. The contribution of Exos to periprosthetic osteolysis was evaluated performing functional tests stimulating primary human macrophages with bone-derived Exos.

Results

For the first time, we report that $\mathrm{TiO}_{2} \mathrm{NPs}$ enter in multivesicular bodies, the nascent of Exos and altered osteoblasts derived exosomes secretion and cargo. No significant differences were observed in Exos morphology and size, however mass spectrometry analysis identified urokinase-type plasminogen activator (UPA), specifically enriched in Exos derived from bone cells pre-incubated with $\mathrm{TiO}_{2} \mathrm{NPs}$. Functional tests confirmed the activation of human macrophages towards a mixed phenotype with consequent secretion of pro and anti-inflammatory cytokines.

\section{Conclusions}

The external stimuli of osteoblasts to $\mathrm{TiO}_{2}$ NPs induced a dose dependent secretion of Exos, suggesting alterations in their biogenesis as well as in their cargo. Functional tests reveal that enriched uPA exosomal cargo is stimulating macrophages towards a mixed M1 and M2 phenotype inducing the release of pro-and anti-inflammatory signals that are characteristic of periprosthetic osteolysis. Interestingly, uPA may be proposed, in the future, as a possible candidate biomarker to early diagnose particle induced periprosthetic osteolysis, since uPA was also detected in the pseudocapsular interface around implants of patients with loosening of total hip prosthesis and joint replacement surgery, suggesting their active role in disease progression.

\section{Background}


Titanium (Ti) and its alloys are the biomaterials mostly used in joint replacements due to their excellent biocompatibility [1-2]. However, one of the main drawbacks to Ti is its susceptibility to both mechanical wear (due to cyclic loading) and corrosion (due to the contact with biological fluids) events resulting in the release of wear debris and corrosion products that give rise to local and systemic complications to patients [3-5]. Histopathological examinations reveal that Ti wear debris with a diversity of sizes and crystal structures accumulate in the synovia-like peri-implant membrane, bone marrow as well as periimplant regions that are affected by fibrotic and inflammatory changes [6]. Due to their high persistency (low solubility), the interaction of wear debris with cells and tissues induce a complex immune response that can lead to periprosthetic osteolysis (degeneration of bone causing aseptic loosening) and implant failure with a consequent need of a revision surgery [3-5, 7-10]. Besides the severe consequences for the patients' health conditions (e.g., higher rates of mortality, hospital readmission, dislocation, and infection), revision surgeries pose a high financial burden on healthcare systems (longer surgery time, expensive prostheses, longer hospitalization periods, and higher rates of health complications) [10-11].

The main biological mechanisms underlying the response to Ti wear debris are innate immune response where the phagocytosis of wear particles induces macrophages activation [12]. The reactivity of macrophage results in a pro-inflammatory milieu (up-regulation of pro-inflammatory cytokines, mainly IL$1 \mathrm{~b}, \mathrm{IL}-6$, and TNF-alfa) in the proximity of bone that perturb its homeostasis (reduced osteoblastogenesis and increased osteoclastogenesis) leading to periprosthetic bone osteolysis [5, 7-10, 12]. Although chronic inflammatory reaction is mostly driven by macrophages, osteolysis is also indirectly caused by the contribution of other cell types such as osteoblasts, dendritic cells, osteoclasts, and synovial fibroblasts. The role of osteoblasts in the development of periprosthetic osteolysis has not been fully explored, although they may not be the major cell players, they play an essential synergetic role by coordinating macrophages and osteoclasts behaviour. Thus, elucidating the response of osteoblasts to wear debris is critical to obtain an understanding of osteolysis as a whole. Besides that, chronic inflammation can be maintained by a continuous release and diffusion of soluble mediators that drive progressive bone resorption, but it can also cause severe and extended pathologies such as metallosis or necrotic-appearing soft tissue masses [13]. One red flag is the ability of some debris, especially those at the nanoscale, to cross epithelial barriers and travel through the lymphatic or circulatory system and accumulate far from the site of the implant resulting in systemic toxicity [3-5]. Their circulation protected from phagocytosis by patrolling monocytes can be supported by extracellular vesicles (e.g. exosomes (Exos)) which are central players in intracellular communication [14-15]. Extracellular vesicles can be found in all body fluids carrying a variable spectrum of molecules characteristic of their cells of origin, with potential to alter the function and physiology of recipient cells. Exos regulate many pathophysiological processes including immune and inflammatory responses, carrying their enriched content (miRNAs, DNA, peptides), and can be found in circulation as a source of biomarkers [14]. We believe that besides the internal response of osteoblasts to the uptake of wear debris, the cell communication with their tissue background can be altered, where Exos can play an important and active role. The effect of Ti nanodebris on bone and immune cell communication is lacking, and the detailed mechanisms underlying Exos biological contribution to osteolysis remain unknown. Thus, in this work we 
start by exploring the effect of titanium dioxide nanoparticles $\left(\mathrm{TiO}_{2} \mathrm{NPs}\right)$ mimicking wear debris released from prosthetic devices on bone derived Exos biogenesis and their involvement in inflammatory responses that are considered as the hallmark of osteolysis. As a proof-of-concept, we investigate the effect of $\mathrm{TiO}_{2}$ NPs on osteoblasts-derived Exos secretion using two human osteoblastic-like cell lines with different degrees of maturation widely used in bone research. Exos were the focus of this study since they are widely known to be a way for cells to get rid of unneeded or unwanted materials (such cellular contents and $\mathrm{TiO}_{2} \mathrm{NPs}$ ), they contribute to the promotion of both innate and adaptative immunity but also there are some evidences suggesting Exos as mediators of chemical toxicity [16-18]. For the first time, we reported that $\mathrm{TiO}_{2}$ NPs internalization alters osteoblasts Exos biogenesis and cargo.

Interestingly, exosomal cargo stimulated human macrophages towards a mixed phenotype with consequent secretion of inflammatory cytokines that are described to contribute to periprosthetic inflammation and consequent osteolysis. We believe that studies revealing the precise composition of Exos cargo may reveal revolutionary and transformative information regarding systemic diffusion of toxic signals by orthopaedic wear debris.

\section{Methods}

Characterization of $\mathrm{TiO}_{2} \mathrm{NPs}$ (mimicking nano wear debris): The dry powder of $\mathrm{TiO}_{2} \mathrm{NPs}$ (Product No. 1317-70-0, particle size $<25 \mathrm{~nm}$ and surface area: $45-55 \mathrm{~m}^{2} / \mathrm{g}$ ) (Sigma-Aldrich). A stock suspension of $\mathrm{TiO}_{2} \mathrm{NPs}$ was prepared in ultrapure water (concentration $2 \mathrm{mg} / \mathrm{mL}$, $\mathrm{pH}$ 4). The samples were dispersed in an ultrasound (ultrasound, Q-Sonica) equipped with a $19 \mathrm{~mm}$ Ti tip. The sonication was performed at 32 W of power for 15 minutes (min) in pulse mode (8 second (sec) ON and 2 sec OFF). Particle size distribution analysis was dynamic light scattering (DLS) using a ZetaSizer Nano ZS (Malvern Instruments) after 24 hours (h) of stabilization. The characterization of $\mathrm{TiO}_{2} \mathrm{NPs}$ in the cell culture medium was performed by diluting the suspension of $2 \mathrm{mg} / \mathrm{mL}$ of NPs in Minimum Essential Medium (aMEM, Gibco) supplemented with $10 \%$ fetal bovine serum (V/V) (FBS, Gibco), pH 7.4. The characterization and dispersion of $\mathrm{TiO}_{2} \mathrm{NPs}$ have been previously published [19-20]. All the suspensions were also characterized by high-resolution transmission electron microscopy (HRTEM, JEOL $2100 \mathrm{~F}$ operating at 200 kV equipped with an X-ray Detector (EDX - Energy-dispersive X-ray spectroscopy)).

\section{MG63 and SAOS-2 cell viability, proliferation, internalization upon nanoparticle exposure}

The cells (MG63 and SAOS-2) were exposed to $\mathrm{TiO}_{2} \mathrm{NPs}(100 \mu \mathrm{g} / \mathrm{mL})$ for $72 \mathrm{~h}$. After viability, it was measured using $2 \times 10^{5} \mathrm{cell} / \mathrm{mL}$ using the Annexin V Dead Cell Apoptosis Kit (Life and Dead Kit, Life Technologies) in a flow cytometer (FACSAria III, BD Biosciences). The procedure is identical to the one previously reported. The analysis was repeated in three independent experiments. The internalization of NPs in MG63 and SAOS-2; all samples were fixed with modified Karnovsky for $2 \mathrm{~h}$ at room temperature and washed with $0.1 \mathrm{M}$ cacodylate buffer with $1 \%$ uranyl acetate (diluted in water) (uranyl acetate, Sigma-Aldrich) overnight. The samples were dehydrated in a series of ethanol (Ethanol, VETEC) (30$100 \%)$ and finally included in Epon 812 resin (EMS). Ultrathin sections (70 nm) were cut in 
ultramicrotome and examined under transmission electron microscopy (TEM, Tecnai Spirit G2, FEI) and a three-dimensional reconstruction was performed using the focused ion beam (FIB) technique. At least ten cells from each group (control and $100 \mu \mathrm{g} / \mathrm{mL}$ ) were analyzed. For proliferation assay, cells were plated $\left(3 \times 10^{4} \mathrm{cell} / \mathrm{cm}^{2}\right)$ in 24-well plates (Corning) and after NPs exposure, cells were washed 3 times with PBS $0.01 \mathrm{M}$ and then incubated in $0.1 \%$ Triton X-100 (TX-100, Sigma-Aldrich) for $10 \mathrm{~min}$. The DNA was quantified by the PicoGreen ${ }^{\circledR}$ dsDNA quantification assay kit (PicoGreen ${ }^{\circledR}$, Invitrogen) according to the manufacturer's instructions. Fluorescence of the plate was read on a microplate reader (Synergy HT, Bioek) using excitation at $480 \mathrm{~nm}$ and emission at $520 \mathrm{~nm}$.

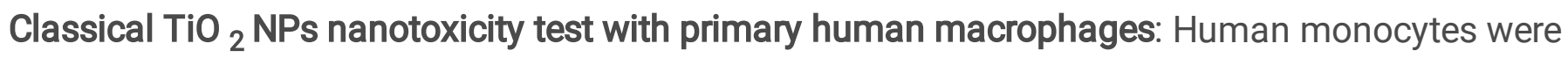
isolated from buffy coats from healthy blood donors, as previously described [21]. Briefly, buffy coats were centrifuged for $30 \mathrm{~min}$, at $1200 \mathrm{~g}$, without brake. The whitish layer containing peripheral blood mononuclear cells was collected and incubated with the RosetteSep Human Monocyte Enrichment Cocktail (StemCell Technologies), during 20 min and under rotation, following the manufacturer's instructions. This mixture was diluted (1:1) in phosphate- buffered saline (PBS $0.01 \mathrm{M}$ ) supplemented with 2\% FBS (Biowest), added over Histopaque-1077 (Sigma-Aldrich) and centrifuged as previously described. The intermediate layer, enriched in monocytes, was collected and washed three times in PBS $0.01 \mathrm{M}$ and centrifuged at 1300 r.p.m. for $6 \mathrm{~min}$. On average, $90 \%$ of isolated monocytes were found to be CD14-positive. For monocyte-macrophage differentiation, $1 \times 10^{6} \mathrm{cell} / \mathrm{cm}^{2}$ monocytes were seeded on circular glass coverslips with $30 \mathrm{~mm}$ diameter (6-well plates, Marienfeld), in complete RPMI medium (10\% FBS and 1\% Penicillin/Streptomycin (Gibco)), supplemented with $50 \mathrm{ng} / \mathrm{mL}$ rhM-CSF (Immunotools), for 7 days. Then, the medium was renewed without rhM-CSF supplementation for additional 3 days. On day 10 , the macrophages were treated with $\mathrm{TiO}_{2} \mathrm{NPs}(100 \mu \mathrm{g} / \mathrm{mL})$ or with different concentrations of Exos (4 $\times 10^{3}, 4 \times 10^{5}$ and $4 \times 10^{7}$ Exos/mL (obtained from MG63 with and without contact with $\mathrm{TiO}^{2} \mathrm{NPs}$ )) and incubated at $5 \% \mathrm{CO}_{2}$, at $37^{\circ} \mathrm{C}$ for additional $72 \mathrm{~h}$. Human monocytes were isolated from buffy coats from healthy blood donors, obtained at Centro Hospitalar Universitário São João (CHUSJ). All studies using these human samples were approved by CHUSJ Ethics Committee for Health (References 259 and 260/11), in agreement with the Helsinki declaration. Informed consent was obtained from all subjects.

\section{Macrophage cytokine profile activation}

Following treatment with $\mathrm{TiO}_{2} \mathrm{NPs}$ and MG63 Exos (as described above), the human macrophage culture supernatant media was collected. Media were collected and centrifuged at $1200 \mathrm{rpm}$ for $5 \mathrm{~min}$ to remove cell debris. The concentration of IL-6, IL-10, IL-1b, TNF-a, IFN-g and TGF-b was determined by ELISA (Biolegend), according to the manufacturer's instructions. As a control were also evaluated in media without addition of NPs.

\section{Macrophage viability}

Flow cytometry was used to measure cell viability. $5 \times 10^{5}$ cell/ $\mathrm{mL}$ were analyzed using the Annexin $\mathrm{V}$ Dead Cell Apoptosis Kit (Life and Dead Kit, Life Technologies), according to manufacturer's instructions 
(through the combination of annexin $\mathrm{V}$ and propidium iodide (PI), it is possible to distinguish the \% viable

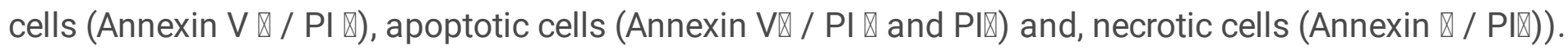
The analysis was performed with macrophages from at least 4 different donors. Cells were acquired on a FACSCanto flow cytometer (BD Biosciences) and analyzed with FlowJo software (v10.6.1).All analyzes were performed on a flow cytometer (FACSAria III, BD Biosciences).

\section{Macrophage morphology and cytoskeleton evaluation}

Upon NPs or Exos' treatments, macrophages were fixed with 4\% paraformaldehyde, quenched with 50 $\mathrm{mM} \mathrm{NH}_{4} \mathrm{Cl}$ for 10 min and, after PBS $0.01 \mathrm{M}$ washes, permeabilized during 5 min with $0.1 \%$ Triton X-100. Blocking was performed for 30 min with $5 \%$ bovine serum albumin (Sigma-Aldrich). Coverslips were incubated for $1 \mathrm{~h}$ with monoclonal anti-a-tubulin (Sigma-Aldrich) antibody, washed and incubated, for an additional $45 \mathrm{~min}$, with goat anti-mouse AlexaFluor-488-conjugated-secondary antibody (ThermoFisher Scientific). F-actin was stained with Alexa Fluor 569 Phalloidin (ThermoFisher Scientific) for 20 min. Coverslips were washed and mounted with Vectashield + DAPI (VectorLaboratories). Cell morphology was analyzed under a Leica TCS-SP5 AOBS spectral confocal microscope (Wetzlar, Germany).

Macrophage surface markers: For the flow cytometry analysis of cell surface receptor expression, upon NPs or Exos treatments, macrophages were detached by incubation with Accutase (Grisp) at $37^{\circ} \mathrm{C}$ for 30 min and harvested by gentle scraping. The cells were washed and resuspended in FACS buffer (PBS 0.01 $\mathrm{M}, 2 \% \mathrm{FBS}, 0.01 \%$ sodium azide) and stained with specific conjugated fluorophore-antibodies, in the dark, for 45 min at $4^{\circ} \mathrm{C}$. Macrophages were incubated with the following antibodies: anti-human CD14-APC (clone MEM-18; Immunotools), CD163-PE (clone GHI/61; R\&D Systems) and CCR7-PerCPCy5.5 (clone $\mathrm{G043H7}$; Biolegend). Isotype-matched antibodies were used as negative controls. Cells were acquired on

a FACSCanto flow cytometer (BD Biosciences) and analyzed with FlowJo software (v10.6.1). The median fluorescence intensity (MFI) was calculated by subtracting the respective isotype control intensity. Data was obtained with macrophages from at least 3 different donors.

\section{Reactive oxygen species quantification}

The identification of reactive oxygen species (ROS) was performed using the 2,7-dichlorofluorescein diacetate probe (H2D-CFDA). Cells were incubated (in samples interacted with $\mathrm{TiO}_{2} \mathrm{NPs}$ ) with 2,7dichlorofluorescein at an ambient concentration of $10 \mu \mathrm{M}$ in the dark (10 min) using protocols provided by the manufacturer (Molecular Probes). Cultures were photographed on an inverted phase contrast microscope (Nikon TMS). They were quantified by measuring the fluorescence intensity measured at the wavelengths of $488 \mathrm{~nm}$ and $530 \mathrm{~nm}$ in a microplate reader (Synergy HT, BioTek). The analysis was repeated in 3 independent experiments.

\section{Macrophage lysosome staining}

Lysosomes (in samples interacted with $\mathrm{TiO}_{2} \mathrm{NPs}$ ) were stained with $500 \mathrm{nM}$ lysotracker (Life) (green) in culture medium for $15 \mathrm{~min}$ at $37^{\circ} \mathrm{C}$ (following kit recommendations) and were incubated with DAPI 
(Sigma-aldrich) for core labeling. Cultures were photographed on an inverted phase contrast microscope (Nikon TMS).

\section{Macrophage $\mathrm{TiO}_{2} \mathrm{NPs}$ internalization}

The internalization of $\mathrm{TiO}_{2}$ NPs in macrophage were evaluated as previously described.

Isolation and characterization of bone derived-exosomes: The minimal essential medium (a-MEM, Gibco) supplemented with $10 \%$ fetal bovine serum (V/V) (FBS, Gibco) was used for the culture of osteoblasts: MG63 (human pre-osteoblasts) and SAOS-2 (osteoblasts) mature humans). Exos-free fetal bovine serum was used in the experiments to obtain Exos [22]. Human osteoblast cell lines were supplied by the Rio de Janeiro Cell Bank (BCRJ), where they were packed in freezer vials and kept in liquid nitrogen. After thawing, the cells were expanded into 25 and/or $75 \mathrm{~cm}^{2}$ cell culture flasks (Corning). The cells used in the experiments were between $2^{\circ}$ and $3^{\circ}$ and were kept in a humidified incubator $\left(5 \% \mathrm{CO}_{2}, 37^{\circ} \mathrm{C}\right)$.

Contamination of cells with bacteria, fungi and mycoplasma was analyzed.

\section{Isolation of exosomes from MG63 and SAOS-2}

To obtain Exos, $5 \times 10^{5}$ cells $/ \mathrm{cm}^{2}$ (MG63 and SAOS-2) were cultured in culture medium supplemented with $10 \%$ FBS (Exos-free) for $72 \mathrm{~h}$. Cells were washed 3 times with PBS $0.01 \mathrm{M}$ and the medium was collected and centrifuged at $4000 \mathrm{xg}, 4$ for $10 \mathrm{~min}$ (Sigma). The supernatant was collected and filtered with $0.2 \mu \mathrm{m}$ filters (Corning) and transferred to pollinator (Beckman) ultracentrifugation tubes (Beckman Optima L80-XP) using the SW41 rotor (Beckman) for $16 \mathrm{~h}$ at 100,000 $\mathrm{g}$ at $4^{\circ} \mathrm{C}$ (Beckman Optima L80-XP) using the SW41 rotor (Beckman) for $6 \mathrm{~h}$ at 100,000 g. Exos were then washed, where the pellet was resuspended in $1 \mathrm{~mL}$ of PBS $0.01 \mathrm{M}$ and another ultracentrifugation was performed (Beckman Optima L80-XP). The supernatant was removed completely, and the Exos pellet was resuspended in $100 \mu \mathrm{L}$ of PBS $0.01 \mathrm{M}$. The same procedure with the same number of cells $\left(5 \times 10^{5} \mathrm{cell} / \mathrm{cm}^{2}\right)$ were performed after the interaction of the cells (MG63 and SAOS-2) with the $\mathrm{TiO}_{2} \mathrm{NPs}(5,10,25,50$ and $100 \mu \mathrm{g} / \mathrm{mL}$ ) for $72 \mathrm{~h}$. To remove the high number of NPs in the supernatants, the cells were washed 3 times with PBS $0.01 \mathrm{M}$ and a fresh culture medium was added with $10 \%$ FBS (Exos-free). After $24 \mathrm{~h}$, the medium was collected and centrifuged as previously described. As controls, cell supernatants were used without interaction with NPs. To eliminate any trace of NPs, a sucrose gradient was performed on the Exos isolates from both cell models.

Purification of exosomes: Sucrose Gradient: Purification of Exos was performed with a continuous sucrose gradient following the protocol already described [22]. Briefly, the Exos were resuspended in $2 \mathrm{~mL}$ of $2.5 \mathrm{M}$ sucrose, $20 \mathrm{mM} \mathrm{HEPES} / \mathrm{NaOH}, \mathrm{pH}$ 7.2. A linear gradient of sucrose $(0.25 \mathrm{M}-2.0 \mathrm{M}$ sucrose, $20 \mathrm{mM}$ $\mathrm{HEPES} / \mathrm{NaOH}$ ) was layered on top of the Exos suspension in an SW41 tube (Beckman). Gradients were centrifuged for $16 \mathrm{~h}$ at $210,000 \times \mathrm{g}$ and $1 \mathrm{~mL}$ fractions were collected from the top of the tube. The densities were evaluated using a refractometer. Exos have been found to float at densities ranging from 1.15 to $1.19 \mathrm{~g} / \mathrm{mL}$ on continuous sucrose gradients [22]. 
Nanoparticle Trace Analysis (NTA): Malvern NTA (NanoSight NS300) was used to measure the size distribution and concentration of intact Exos isolated from cultures of MG63 and SAOS-2. The isolated Exos were resuspended in PBS $0.01 \mathrm{M}$ (diluted 3:1000) and analyzed. The protocol applied was previously described [22].

Identification of $\mathrm{TiO}_{2}$ NPs in exosome isolates by - Inductively Coupled Plasma Mass Spectrometry (ICPMS): The presence of $\mathrm{Ti}$ (titanium) in solution of purified Exos and/or not by sucrose gradient (Exos obtained after treatment of $50 \mu \mathrm{g} / \mathrm{mL}$ and $100 \mu \mathrm{g} / \mathrm{mL}$ ) was investigated. The positive control was the stock solution of $\mathrm{TiO}_{2} \mathrm{NPs}(2 \mathrm{mg} / \mathrm{mL}$ ) and the negative control was PBS $0.01 \mathrm{M}$ (without contact with NPs). Titanium (48Ti) analysis was performed using an iCAP $^{\text {TM }} \mathrm{Q}$ instrument (Thermo Fisher Scientific, Bremen, Germany), equipped with a Meinhard ${ }^{\circledR}$ TQ + high-sensitivity nebulizer, a disconcerted (Peltiercooled) cyclonic spray chamber, a Standard quartz torch and a two-cone design (nickel sample and skimmer cones). High purity argon (99.9997\%) (Gasin, Portugal) was used as a nebulizer and as a source of plasma gas. The operating parameters of the ICP-MS instrument were as follows: RF power (1550 W); plasma gas flow $(14 \mathrm{~L} / \mathrm{min})$; auxiliary gas flow $(0.8 \mathrm{~L} / \mathrm{min})$; nebulizer flow rate $(1.01 \mathrm{~L} / \mathrm{min})$. Elemental isotope scandium (45Sc) was used as the internal standard. Samples were diluted 1:20 and their concentrations were derived from linear regression equations representing the relationship between the analytical signal (ICPS) and the elemental concentration of the corresponding standard solution.

\section{Transmission Electron Microscopy (TEM) of exosomes}

The Exos isolation and purification by gradient sucrose were applied as described before [22]. To remove aggregates of proteins and improve the morphological analysis of Exos, a wash (PBS $0.01 \mathrm{M}$ ) was performed. Basically, after isolation and purification, Exos were resuspended in PBS $0.01 \mathrm{M}$ and transferred to polyallomer tubes (Beckman) and placed in the ultracentrifuge (Beckman Optima L80-XP) using the SW41 rotor (Beckman), and centrifuged for $16 \mathrm{~h}$ at $100,000 \mathrm{~g}$ at $4^{\circ} \mathrm{C}$. After, Exos were resuspended in $50 \mathrm{uL}$ of PBS $0.01 \mathrm{M}$, and a drop of the suspension was placed onto a holey coated copper grid. Samples were contrasted in $1 \%$ uranyl acetate and visualized in the transmission electron microscope (TEM, Tecnai Spirit G2, FEI).

\section{Protein Analysis by SDS-PAGE and Western Blots}

For identification of the CD63, Exos protein, 20 exos/uL solution (from MG63 and SAOS-2) without interaction with NPs were added to $10 \mu \mathrm{L}$ of $1 \mathrm{x}$ buffer ( $0.0625 \mathrm{M}$ Tris-HCL, $2.5 \% \mathrm{SDS}, 5 \%$ Glycerol) and frozen to $-20^{\circ} \mathrm{C}$. Samples were boiled $10 \mathrm{~min}$ at $100^{\circ} \mathrm{C}$ and run on $7.5 \%$ bis-polyacrylamide gel, $120 \mathrm{~V}$ were used to separate the proteins by molecular weight. Therefore, the gel was stained using $1 \%$ colloidal Coomassie blue (BIO-RAD). The bis-polyacrylamide gel was transferred to nitrocellulose membrane (Millipore) at $120 \mathrm{~V}$ for 120 min. Membranes were blocked in PBS/0.1\% Tween-20 (PBS/T) with 5\% skim milk, incubated with CD63 primary antibody diluted in PBS/T (Mouse - Novusbio), incubated overnight. The membranes were washed four times in blocking solution, and finally incubated with conjugated secondary antibody (anti-CD63, Novusbio) followed by washing in PBS/T. Blots were developed using the 
ECL Plus (GE Healthcare) Western Blotting Detection System accordance with the manufacturer's instructions.

\section{Identification of proteins present in exosomes by mass spectrometry}

The proteins present in Exos isolated from human osteosarcomas (MG63 and SAOS-2) were analyzed. To this end, we investigated the proteins present in Exos samples obtained from control without contact with $\mathrm{TiO}_{2} \mathrm{NPs}$ of both cell models and in samples obtained after interaction with $\mathrm{TiO}_{2} \mathrm{NPs}$ for $72 \mathrm{~h}$. Samples were purified by sucrose gradient and washed to remove contaminating proteins. The concentration of Exos was normalized for all points (about $90 \mu \mathrm{g} / \mathrm{mL}$ protein at each point analyzed). An enzymatic digestion was then performed using $0.2 \mu \mathrm{g}$ trypsin (Promega) diluted in ammonium bicarbonate (50 Mm, 30 min in overnight ice at $37^{\circ} \mathrm{C}$ ). The extracted peptide mixture was lyophilized in $1 \%$ formic acid and transferred to StageTip (C18). A drying process was applied. Then $25 \mu \mathrm{L}$ of methane acid (1\%) (Sigma) was added. Samples were analyzed on a mass spectrometer (MS) (EDT-enabled Orbitrap Velos) (ThermoFisher scientific) coupled to an EASY-nLC (Proxeon Biosystem) system using a Proxeon nanoelectrosplay source. The peptides were separated on a gradient of $2-90 \%$ acetonitrile in $1 \%$ methane acid in a PicoFrit analytical column $(20 \mathrm{~cm} \times$ ID75 $\mu \mathrm{m}, 5 \mu \mathrm{m}$ particle size), with a flow of $300 \mathrm{~nL} / \mathrm{min}$ for $27 \mathrm{~min}$. The nanoelectrosplay voltage and temperature were adjusted to $2.2 \mathrm{kV}$ and $275^{\circ} \mathrm{C}$, respectively. The method configured for LTQ Orbitrap Velos was data dependent analysis (ADD). SM scanning spectra ( $\mathrm{m} / \mathrm{z} 300-$ 1600) were acquired on the Orbitrap analyzer after accumulation to a target value of 1 and 6 and the Orbitrap resolution was adjusted to $r=60,000$. Thus, the 20 most intense peptide ions with charge states $\geq 2$ were sequentially isolated to a target value of 5,000 and fragmented into the linear low energy CID ion trap ( $35 \%$ normalized collision energy). The signal threshold for triggering an SM/SM event has been set to 1,000 counts. Dynamic deletion was enabled with a size list of 500 and the deletion duration was $60 \mathrm{sec}$. The activation Q value was 0.25 and the activation time was $10 \mathrm{~ms}$. Data were obtained using the Xcalibur software package and the samples were analyzed in three biological replicates. Peak lists (msf) were generated from files containing raw data using Proteome Discoverer version 1.3 (Thermo-Fisher scientific) with the Sequest search engine and searched against taxon $\mathrm{H}$ sapiens from the UniProtKB/SwissProt database (release 2016_04) with carbamidomethylation as a fixed modification. The Software (version Scaffold_4.5.1, Proteome Software Inc., Portland, OR) was used to validate SM/SM-based peptide and protein identifications. Peptide identifications have been accepted when a probability greater than $99.0 \%$ can be established to achieve an FDR of less than $1.0 \%$ by the Scaffold local FDR algorithm. Protein identifications were accepted when a probability greater than $80.0 \%$ could be established to achieve an FDR less than $1.0 \%$ and contained at least one identified peptide. Protein probabilities were assigned by the Protein Prophet algorithm. Proteins that contained similar peptides and could not be differentiated based on the SM / SM analysis alone were pooled. Proteins were noted with GO terms of $\mathrm{H}$ sapiens filtered (gene_association.goa_cow.gz, downloaded 06-Apr-2016). Considering the control versus treatment ( $\mathrm{p} 0.01$ ) we obtained the proteins that increased and decreased after the treatment of $\mathrm{TiO}_{2} \mathrm{NPs}$ in the studied cells. Results are the mean \pm standard deviation of triplicate independent experiments. 


\section{Statistical analysis}

Data were presented as mean \pm standard deviation $( \pm S D)$. Gaussian distribution of samples was tested, and the statistical significance of the data was evaluated using One-way ANOVA and unpaired t-test was applied to obtain statistical significance of means. The $P$ value is shown in figures and statistical significance was considered when $p<0.05$. Each experiment was performed in three independent experiments with triplicates.

\section{Results And Discussion}

\section{Osteoblast-derived exosomes exposed to nano-wear debris alter the cytokine profile secreted by human macrophages}

The gradual inflammatory response to titanium implant-derived wear particles is the hallmark of periprosthetic osteolysis that is illustrated by an innate immune response [16-17, 23]. This response is characterized by macrophage reactivity to implant wear debris, resulting in pro-inflammatory cytokines, chemokines and pro-osteoclastic factors release that leads to an increased osteoclastogenesis with subsequent bone resorption around the implant (periprosthetic osteolysis) [17, 23-24]. Besides macrophage activation in order to eliminate implant nano-debris that is driven by the chemical and the physical features of the particles, several others cells types including osteoblasts, monocytes, fibroblasts, osteoclasts, and mesenchymal stem cells present at the interface protheses-bone are affected and contribute to osteolysis [23-24].

We have previously reported that $\mathrm{TiO}_{2} \mathrm{NPs}$, similar in size to wear debris associated with prosthetic implants, were internalized by primary human osteoblasts inducing the release of pro-inflammatory cytokines. Results that support the direct involvement of osteoblastic cells in inflammatory processes in response to wear debris [19-20]. However, at the implant-bone interface there is a series of differentiation events occurring during bone healing, where debris enter in direct contact with mature and immature osteoblasts among other cells. To understand how osteoblasts with different degrees of differentiation behave in face of nano-debris exposure, MG63 and SAOS-2 cell lines were used and stimulated with 100 $\mu \mathrm{g} / \mathrm{mL}$ of $\mathrm{TiO}_{2} \mathrm{NPs}$ during $72 \mathrm{~h}$. $\mathrm{TiO}_{2} \mathrm{NPs}$ revealed a round-shape like morphology with a primary size of $20 \mathrm{~nm}$ (Fig. 1A). As it can be seen, in medium culture, $\mathrm{TiO}_{2}$ NPs agglomerate in structures of about 140 $\mathrm{nm}$ with specific proteins and ions adsorbed on them (Fig. 1B), these being the main events occurring at the nano-biointerface, as already reported [20]. The main physicochemical characteristics of $\mathrm{TiO}_{2} \mathrm{NPs}$ are presented in Fig. 1C. After $\mathrm{TiO}_{2}$ NPs characterization, osteoblasts with different degrees of maturation were exposed to $\mathrm{TiO}_{2} \mathrm{NPs}$ and Exos isolated, following the workflow represented in Fig. 1D.

Our data demonstrated that $\mathrm{TiO}_{2}$ NPs did not compromise osteoblasts viability and proliferation, confirming that bone cells with diverse degrees of differentiation react in a similar way to $\mathrm{TiO}_{2} \mathrm{NPs}$ (Fig. 2A-D). Interestingly, in both cell lines, $\mathrm{TiO}_{2} \mathrm{NPs}$ were preferentially located inside multivesicular 
bodies (MVBs), the nascent of Exos (Fig. 2E, F and G). This finding indicates that NPs in endosomes maturation can proceed in two independent pathways: i) fusion of $\mathrm{TiO}_{2} \mathrm{NPs}$-containing MVBs with lysosomes, where acid compartments ensure degradation of their content (although $\mathrm{TiO}_{2} \mathrm{NPs}$ are not degraded due to its physicochemical characteristics); ii) MVBs can also fuse with the plasma membrane, releasing their contents as Exos in the extracellular space [13-15, 25-26]. High resolution images and 3D reconstruction obtained by focusing ion beam, reveal $\mathrm{TiO}_{2} \mathrm{NPs}$ isolated in multivesicular bodies (MVBs) in direct contact with Exos (Fig. 2G).

A detailed description of the cellular and the molecular mechanisms underlying the biological response to implant debris is widely described in literature $[1-2,4,9,11,23]$. Accumulating evidence has revealed the roles of osteoblast-derived Exos in mediating intercellular crosstalk in bone remodeling, however the possible contribution of Exos cargo to the systemic diffusion of toxic signals and activation of innate immune activation is lacking [27]. Taking that in consideration, we next questioned if $\mathrm{TiO}_{2} \mathrm{NPs}$ could affect osteoblast-derived Exos biogenesis and affect osteoimmune crosstalk. To assess the influence of $\mathrm{TiO}_{2} \mathrm{NPs}$ on bone-immune cells crosstalk, functional assays were performed exposing different amounts of Exos derived from MG63 bone cells that were pre-incubated (ExoMG63 + NPs_1:3) or not (ExoMG63_1:3) with $100 \mu \mathrm{g} / \mathrm{mL} \mathrm{TiO}_{2}$ NPs to primary human macrophages (see the scheme presented in Fig. 3A). Exos were isolated using established ultracentrifugation methods [22] and purified with sucrose gradient to eliminate any trace of $\mathrm{TiO}_{2} \mathrm{NPs}$. Pro-inflammatory (interleukin 6 (IL-6), tumor necrosis factor a (TNF- $\alpha$ ), interleukin 1 beta (IL-1 $\beta)$ ) and anti-inflammatory (interleukin 10 (IL-10), transforming growth factor beta (TGF- $\beta$ )) cytokines, macrophage viability, cytoskeleton organization and polarization surface markers were evaluated. Excitingly, we observed that the higher concentration of osteoblast-derived Exos (ExoMG63 + NPs_3) induced a significant increase of human macrophage secretion of both IL-6 and IL10 (Fig. 3B and Figure in additional files 1). Individual cytokine results presented by donors are shown in Figure in additional files $1 \mathrm{~A}$. These results indicate that Exos-derived from osteoblasts cells treated with $\mathrm{TiO}_{2}$ NPs specifically induced macrophage activation unlike their counterparts (ExoMG63_1:3). Innate immune implant debris-induced inflammation is caused predominantly by macrophages, that together with inflammatory micro-environmental signals induce an M1-like macrophage polarization, exacerbating the production of inflammatory signals (IL-8, monocyte chemotactic protein-1 (MCP-1), macrophage inflammatory protein 1 (MIP-1), IL-6, IL-1 $\beta$, TNF-a) that favors osteoclastogenesis with consequent osteolysis and prothesis failure [9, 28-31]. Not only M1 macrophages release inflammatory mediators and catabolic enzymes that drive the inflammatory process, M2 macrophages also produce TGF- $\beta$, IL10 and other signaling molecules with the goal of resolving inflammation and inducing tissue regeneration $[30,32]$. Detailed microscopic analysis revealed alterations on macrophage morphology (Fig. 3C and Figure in additional files 2), a known indicator of macrophage phenotype/activation status [21], as evidenced by the alterations between $\mathrm{TiO}_{2} \mathrm{NPs}$-treated and untreated macrophages (control). In parallel, Exos-derived from osteoblasts pre-incubated with $\mathrm{TiO}_{2} \mathrm{NPs}$ induced an overall increase in cell area and a less intense cortical actin ring. Specially in the intermediate Exos concentration, a shift on morphology 
was clearly observed, with a higher density of fusiform macrophages, typical of an M1-like phenotype, as previously described [21]. Despite this, Exos did not alter the viability of macrophages (Fig. 3D).

No significant differences were observed on IL-1 $\beta$, TNF- $\alpha$ or TGF- $\beta$ secretion levels (see Figure in additional files 1B) neither on the expression of macrophage phenotype-associated surfaces markers (Fig. 4 and Figure in additional files 3), which could be most likely explained by the existence of a mixed M1-M2 macrophage phenotype (as it happens at the implant site), and also to the inherent variability between blood donors. Macrophage polarization in joint tissues remains controversial. Although it has been reported that particle-induced response is prone to drive macrophages towards M1 phenotype, in some studies, the density of metallic particles on aseptic interface tissues did not lead to local preferential M1 macrophage polarization [12]. Clinical studies reveal that in the end-stage osteolysis is characterized by an unconventional macrophage activation pathway within periprosthetic tissues that is distinguished by generation of a specific cytokine and chemokine milieu, but not by elevated levels of proinflammatory cytokines [33]. It is possible that the pro-inflammatory responses may be transient in nature, with M2 responses being supplanted in the later stages of disease progression [33-34].

Comparing the effect of osteoblast-derived Exos with direct contact of $\mathrm{TiO}_{2} \mathrm{NPs}$ on the behavior of macrophages, it was possible to observe comparable results with exception of macrophage viability (Fig. 5A), which was directly compromised by ROS (Fig. 5B), overproduction and increased lysosomal activity (Fig. 5C) induced by $\mathrm{TiO}_{2}$ NPs internalization (Fig. 5D). The decline in macrophage viability after treatment with NPs is probably due to their phagocytic role, causing high levels of internalized NPs, which in turn enter the lysosomal pathway, dramatically increasing ROS levels [12, 34-35]. This induction generates oxidative stress, which will lead to DNA/chromosomal damage and induce death and apoptosis [36].

In sum, our data indicates that Exos derived from pre-osteoblast pre-incubated with $\mathrm{TiO}_{2} \mathrm{NPs}$ modulate macrophages towards a mixed inflammatory profile that has been reported in particle-induced osteolysis and metallosis cases [3].

\section{Nano wear debris alter osteoblasts derived exosomes biogenesis and cargo}

We demonstrated that the identified macrophage activation was a result of Exos. We have used sucrose gradient to isolated Exos from ExosMG63/ExosSAOS-2 and ExosMG63 + NPs/ ExosSAOS-2 + NPs. The isolated Exos express the CD63 marker, as shown by western blot analysis (Fig. 6A), they have the expected Exos diameter $(75 \pm 5 \mathrm{~nm}$ and $80 \pm 4 \mathrm{~nm})$ measured by nanoSight ${ }^{\circledR}$ nanoparticle tracking analysis and TEM micrographs, respectively (Fig. 6B-G). It is important to refer that the isolation and characterization of Exos followed the Minimal Information for Studies of Extracellular Vesicles ("MISEV") guidelines [37]. The role of osteoblast-derived Exos to maintain bone microenvironment was already described. They are known to facilitate a diverse of intracellular and intercellular signaling cascades that regulates both osteoclastogenesis and osteogenesis [25,38]. An increase of Exos secretion was observed upon $\mathrm{TiO}_{2}$ NPs stimulation in both cell lines as observed in Fig. 6B and D (see Figure in additional files 4A 
and B). Exos are important mediators of cell-cell communication, and their biogenesis and fate can be altered by chemicals [14]. The application of Exos research in toxicology is still in its infancy, however emerging studies already reveal that Exos provide a mechanistic link between inhalation exposures and airway inflammation, and systemic effects $[14,39]$. Here we demonstrate for the first time that nanoparticles can also impact Exos biogenesis. Our data clearly demonstrates that the observed activation of macrophages is exclusive of Exos and not a dual effect of Exos and NPs. No significant differences were observed on Exos diameter when osteoblasts cells were pre-incubated with $\mathrm{TiO}_{2} \mathrm{NPs}$ as evidenced by nanoparticle size analysis results (Fig. $6 \mathrm{C}$ and E). Transmission electron micrographs reveal that Exos populations presented a cup-shaped morphology (Fig. $6 \mathrm{~F}$ and $\mathrm{G}$ ) and that $\mathrm{TiO}_{2} \mathrm{NPs}$ were not able to enter in Exos. Inductively coupled plasma spectroscopy analysis (ICP-MS) of isolated Exos (Fig. 6H and I) confirmed that the use of a sucrose gradient was an efficient method to obtain Exos free of metallic components.

Proteomic profiling of Exos derived from MG63 and SAOS-2 was evaluated by mass spectrometry, 636 and 276 proteins being identified, respectively (Fig. 7A; Figure in additional files 5A). Only 242 exosomal proteins were similar in both models (Fig. 7A). We listed the top 20 proteins exclusively identified in ExosMG63 + NPs (Fig. 7B, left panel) and ExosSAOS-2 + NPs (Fig. 7C, left panel). The most abundant proteins found in Exos derived from MG63 pre-incubated with $\mathrm{TiO}_{2}$ NPs were talin-1, fibronectin, filamin-A, actin cytoplasmic 1 and vinculin. While for SAOS-2 identified protein were heparan sulfate proteoglycan (HSPG), fibronectin (FN), actin cytoplasmic 1, alpha-2-macroglobulin and thrombospondin-1. Gene ontology analysis demonstrated that the most abundant proteins found in both cell models are associated with cell organization and biogenesis, metabolic and regulation of biological processes, response to stimulus, transport (Fig. 7B and C, right panels) and KEGG pathways (Figure in additional files $5 \mathrm{~B}$ and $\mathrm{C}$ ). At the proteome level, the significantly altered cellular components were cytoplasm, cytosol, extracellular region and cellular membrane. Exos shed by pre-osteoblasts and mature osteoblasts were also enriched in cytoskeleton proteins that are among the most abundant components that make up extracellular vesicles, proposing their contribution to Exos formation and/or the regulatory signaling functions. It is possible that as Exos fuse with target cells, the presence of cytoskeletal components on Exos could enhance cellular uptake and cellular responses like direct cell movement [38]. In both cell models, fibronectin (FN) was also detected in Exos cargo. There are multiple evidences suggesting that FN facilitate Exos cell binding, uptake and motility [38 40]. Besides that, FN is also contributing to the induction of the mixed-inflammatory microenvironment observed, since human derived exosomal fibronectin was reported to induce pro-inflammatory cytokines production [21]. Furthermore, fibronectin and cytoskeleton proteins, heparan sulfate (HSPG) were detected on Exos derived from differentiated osteoblasts [38, 41]. HSPG reside on the surface of Exos and are considered as key players in Exos biogenesis and uptake, having a great potential as biomarkers for liquid biopsy of glioblastoma and pancreatic cancer diagnostics [42].

Interestingly, treatment with $\mathrm{TiO}_{2} \mathrm{NPs}$ resulted in the enriched and under-represent of specific exosomal proteins (Fig. 8A and B). In Exos derived from MG63 (Fig. 8A) pre-incubated with $\mathrm{TiO}_{2} \mathrm{NPs}$ there was an 
enriched of urokinase-type plasminogen activator (UPA) and 60s ribosomal protein, plexin-A1, immunoglobulin kappa constant and immunoglobulin heavy constant gamma were under-represented. Urokinase plasminogen activator is an enzyme that catalyzes the conversion of plasminogen to plasmin. From literature it was already reported by immunohistochemistry the localization of uPA in macrophages of periprosthetic tissue that phagocytosed metal, polyethylene, cement particles or accompanying pieces of necrotic bone [43-44]. The expression of the plasminogen activation system by macrophages containing phagocytosed particles suggests undegradable debris as a possible initiator stimulus for a proteolytic activation cascade, which can contribute to loosening of the prosthesis [45]. Interestingly uPA up-regulated was already identified in the pseudo-capsular and interface tissue around implants of patients with loosening of total hip prosthesis [46-47] and in osteoarthritic patients who undergone total joint replacement surgery [44]. Aberrant production of uPA and cytokines (IL-1ß and IL-6) has already been described in patients with osteoarthritis and rheumatoid arthritis, demonstrating the active role of uPA on disease progression [48-49]. Interestingly, UPA, C-telopeptide of type I collagen, IL-6, together with toll-like receptor, lipopolysaccharide-binding protein and myeloid-related protein-14, have been also suggested as serological biomarkers for accurate prosthetic joint osteolysis and infection diagnosis [50]. Moreover, recent in vitro and in vivo findings indicate that the uPA/uPAR system is an active participant in the majority of infection and inflammatory diseases and might serve as a modulator of immunological responses [44, 51-52]. It has been implicated in macrophage adhesion, motility, proliferation, differentiation, invasion and matrix breakdown $[43,52]$. Previous studies have shown that uPA plays essential roles in immune cell response (macrophages chemotaxis), which aids in the elimination of infectious organisms, tissue inflammation, and regeneration [43]. As uPA is also considered as inflammatory macrophages activator [52], it is possibly contributing to the mixed-inflammatory microenvironment observed upon MG63 Exos macrophage stimulus.

The enriched exosomal proteins in SAOS-2 model enriched upon NPs exposure were C-X-C motif chemokine 6, Histone H4, Proteasome, Transketolase and Histone H2B type 2-F. On the contrary, cell migration-inducing, nidogen-2, serine protease 23 and chitinase-3-like protein 1 were under-represent. Most of the identified enriched proteins were involved on immune response (Fig. 8B). Although no functional tests with macrophages were performed with SAOS-2 derived Exos, it was interesting to observe that the proteins comparing both models were dissimilar. Clearly the degree of cell differentiation affects Exos secretion and cargo upon NPs stimulation.

In conclusion, this study provides, for the first time, insights into the capacity of the osteoblast derived Exos exposed by wear debris to stimulate macrophages towards a periprosthetic inflammatory profile. Besides activation of different cell types migration to the site of inflammation, exosomal cargo enriched in UPA is possibly contributing to the generation of pro-and anti-inflammatory signals. Although there is still the need of analyzing clinical samples, these earlier findings are considered as the first steps towards the proposal of exosomal cargo as a new generation of a non-invasive early diagnostic marker for nanoparticle induced osteolysis (Fig. 9). These results are clinically relevant since they reveal, for the first time, that $\mathrm{TiO}_{2}$ nano-debris with its low solubility can accumulate in the multivesicular bodies of 
osteoblast cells affecting Exos biogenesis. Moreover, there is no need of the direct contact of $\mathrm{TiO}_{2}$ nanoparticles to stimulate macrophage-dominant inflammatory responses, since Exos with its specific cargo are inducing a mixed inflammatory profile. Based on our results and the observed cells at the taper interface, it can be suggested that monocytes recruited to the implant site by inflammation or resident macrophages in the surrounding tissue can be activated by osteoblasts derived Exos that were in contact with wear debris. It is important to refer that our data is based on proteomic landscape and efforts are under to focus on the genomic Exos content.

\section{Conclusions}

This study unravels the distinctive influence of $\mathrm{TiO}_{2}$ wear debris in the form of nanoparticles, on osteoblasts exosome secretion and their interplay with immune cells. Nanoparticles were able to enter in multivesicular bodies, the nascent of exosomes of both bone cell lines altering Exos biogenesis. The size, shape and morphology of Exos was similar, however a distinct exosomal (enriched in uPA) cargo was observed upon $\mathrm{TiO}_{2}$ NPs stimulus. Higher concentration of osteoblast-derived Exos enriched in uPA induced macrophages towards a mixed inflammatory profile that has been reported in particle-induced osteolysis and metallosis cases.

\section{Abbreviations}

Titanium dioxide Nanoparticles - $\mathrm{TiO}_{2} \mathrm{NPs}$; Exosomes - Exos; urokinase-type plasminogen activatoruPA; Multivesicular Bodies - MVBs; Human osteosarcoma cell lines - MG63; Human osteosarcoma cell lines - SAOS-2; Interleukin 6 - IL-6; Interleukin 8 - IL-8; Tumor necrosis factor a - TNF-a; Interleukin 1 beta IL-1b; Interleukin 10 - IL-10; Transforming growth factor beta- TGF-b; Monocyte chemotactic protein-1 MCP-1; Macrophage inflammatory protein 1-MIP-1; Fibronectin -FN; Toll-like receptor- TLRs; Lipopolysaccharide-binding protein - LBP; Myeloid-related protein14 - MRP-14; Reactive Oxygen SpeciesROS; Transmission Eletron Microscopy - TEM; Nanoparticle Tracking Analysis - NTA.

\section{Declarations}

\section{Corresponding Author}

Ana R Ribeiro: ana.ribeiro@inl.int

Present Adress: *The author is currently at: Nanosafety group, International Iberian Nanotechnology Laboratory - INL, 4715-330 Braga, Portugal. E-mail: ana.ribeiro@inl.int

\section{Ethical Approval and Consent to participate}

All studies with human cell samples were approved by CHUSJ Ethics Committee for Health (References 259 and 260/11), in agreement with the Helsinki declaration. Informed consent was obtained from all subjects. 


\section{Consent for publication}

Not applicable.

\section{Availability of data and materials}

The datasets used and/or analyzed during this study are available from the corresponding authors on reasonable request.

\section{Competing interests}

SAM and ARR have ownership interests (patents). The other authors declare no competing interests.

\section{Funding}

This work was supported by National Council for Scientific and Technological Development (CNPq) with grants 405030/2015-0, 306672/2016-2, and 467513/2014-7. A R Ribeiro especially thanks to PropesqUnigranrio-FUNADEP Scholarship and Jovem Ciêntista do Nosso Estado award from FAPERJ. J M G thanks Cientista do Nosso Estado award from FAPERJ. SAM laboratory is supported by European Regional Development Fund (ERDF) through COMPETE 2020 - Operacional Programme for Competitiveness and Internationalization (POCI), Portugal 2020, and by FCT - Fundação para a Ciência e a Tecnologia (FCT)/Ministério da Ciência, Tecnologia e Inovação in the framework of the project POCl-010145-FEDER-032189. WSouza specially thanks to scholarship Capes / FTC - 88887.163123 / 2018-00.

\section{Authors' contributions}

A.R. Ribeiro, W. Souza, L.A. Rocha, Maria J. Oliveira, Maria. H. Fernandes, Sonia A. Melo designed the study. W. Souza, S.G. Piperni, Patrícia Cardoso, Carolina Ruivo, Nuno Bastos performed experimental work and analyses. Wanderson de Souza, Sara G. Piperni, Patrícia Cardoso, Maria J. Oliveira, Luís. A. Rocha, José M. Granjeiro, Maria. H. Fernandes, Sonia A. Melo, Ana R. Ribeiro contributed technical supports and discussion. W. Souza, Maria J. Oliveira, Sonia A. Melo and A.R. Ribeiro wrote/edited the manuscript.

\section{Acknowledgements}

Not applicable.

\section{Authors' information}

${ }^{a}$ Directory of Life Sciences Applied Metrology, National Institute of Metrology Quality and Technology, Rio de Janeiro, Brazil. b Postgraduate Program in Biotechnology, National Institute of Metrology Quality and Technology, Rio de Janeiro, Brazil. 'Postgraduate Program in Translational Biomedicine, University Grande Rio, Duque de Caxias, Brazil. di3S-Institute for Research and Innovation in Health, University of Porto, Portugal, Porto, Portugal. ePhysics Department, São Paulo State University Júlio de Mesquita Filho, 
São Paulo, Brazil. ${ }^{f}$ IBTN/Br - Brazilian Branch of the Institute of Biomaterials, Tribocorrosion and Nanomedicine, São Paulo State University, Bauru, São Paulo, Brazil. ${ }^{9}$ Dental School, Fluminense Federal University, Niterói, Brazil. h'Faculty of Dental Medicine, University of Porto, Porto, Portugal. 'LAQV/REQUIMTE, University of Porto, Porto, Portugal

\section{References}

[1]. Fretwurst, T., Nelson, K., Tarnow, D. P., Wang, H. L. \& Giannobile, W. V. Is Metal Particle Release Associated with Peri-implant Bone Destruction? An Emerging Concept. J. Dent. Res. 97, 259-265 (2018).

[2]. Julien Barthes, Sait Ciftci, Florian Ponzio, Helena Knopf-Marques, Liza Pelyhe, Alexandru Gudima, Imre Kientzl, Eszter Bognár, Miklós Weszl, Julia Kzhyshkowska \& Nihal Engin Vrana. Review: the potential impact of surface crystalline states of titanium for biomedical applications. Crit. Rev. Biotechnol. 38, 423-437 (2018).

[3]. Vasconcelos, D. M., Santos, S. G. \& Lamghari, M. Biomaterials The two faces of metal ions: From implants rejection to tissue repair / regeneration. Biomateriais 84, 262-275 (2016).

[4]. Prokopovich, Polina. Interactions between mammalian cells and nano- or micro-sized wear particles: Physico-chemical views against biological approaches. Advances in Colloid and Interface Science, 213, 36-47 (2014).

[5]. Balachandran, Shanoob; Zachariah, Zita; Fischer, Alfons; Mayweg, David;Wimmer, Markus A.; Raabe, Dierk; Herbig, Michael. Atomic Scale Origin of Metal Ion Release from Hip Implant Taper Junctions. Advanced Science, 1903-008 (2020).

[6]. Schoon J, Hesse B, Rakow A, Ort MJ, Lagrange A, Jacobi D, Winter A, Huesker K, Reinke S, Cotte M, Tucoulou R, Marx U, Perka C, Duda GN, Geissler S. Metal-Specific Biomaterial Accumulation in Human Peri-Implant Bone and Bone Marrow. Adv Sci, 3;7(20):2000412 (2020).

[7]. Ryan D. Ross, Youping Deng, Rui Fang, Nicholas B. Frisch, Joshua J. Jacobs, Dale R.

Sumner. Discovery of Biomarkers to Identify Peri-Implant Osteolysis Before Radiographic Diagnosis. $J$ Orthop Res. 36, 2754-2761 (2018).

[8]. Gruber, R. Osteoimmunology: Inflammatory osteolysis and regeneration of the alveolar bone. J Clin Periodontol. 46, 52-69 (2019).

[9]. Howie DW, Neale SD, Haynes DR, Holubowycz OT, McGee MA, Solomon LB, Callary SA, Atkins GJ, Findlay DM. Periprosthetic osteolysis after total hip replacement: molecular pathology and clinical management. Inflammopharmacol 21, 389-396 (2013).

[10]. Adrese M Kandahari, Xinlin Yang, Kevin A Laroche, Abhijit S Dighe, Dongfeng Pan \& Quanjun Cui. A review of UHMWPE wear-induced osteolysis: the role for early detection of the immune response. Bone 
Res 4, 16014 (2016).

[11]. Mitzi S. Laughlin, Emily A. Vidal, Arin A. Drtil, Robin N. Goytia, Vasilios Mathews, Anay R. Patel, Mortality After Revision Total Hip Arthroplasty. The Journal of Arthroplasty, 0883-5403, (2021).

[12]. Scherbart, A.M., Langer, J., Bushmelev, A. et al. Contrasting macrophage activation by fine and ultrafine titanium dioxide particles is associated with different uptake mechanisms. Part Fibre Toxicol 8, 31 (2011).

[13]. Hessvik, N. P. \& Llorente, A. Current knowledge on exosome biogenesis and release. Cell. Mol. Life Sci. 75, 193-208 (2018).

[14]. Colombo, M., Raposo, G. \& Théry, C. Biogenesis, Secretion, and Intercellular Interactions of Exosomes and Other Extracellular Vesicles. Annu. Rev. Cell Dev. Biol. 30, 255-289 (2014).

[15]. Cocucci, E. \& Meldolesi, J. Ectosomes and exosomes: Shedding the confusion between extracellular vesicles. Trends Cell Biol. 25, 364-372 (2015).

[16]. Hughes, C.S., Colhoun, L.M., Bains, B.K. et al. Extracellular cathepsin S and intracellular caspase 1 activation are surrogate biomarkers of particulate-induced lysosomal disruption in macrophages. Part Fibre Toxicol 13, 19 (2015).

[17]. Pieters, Bartijn C. H.; Cappariello, Alfredo; van den Bosch, Martijn H. J.; van Lent, Peter L. E. M.; Teti, Anna; van de Loo, Fons A. J. Macrophage-Derived Extracellular Vesicles as Carriers of Alarmins and Their Potential Involvement in Bone Homeostasis. Frontiers in Immunology, 10, 1901 (2019).

[18]. Mathieu, M., Martin-jaular, L., Lavieu, G. \& Théry, C. Specificities of secretion and uptake of exosomes and other extracellular vesicles for cell-to-cell communication. Nat. Cell Biol. 21, (2019).

[19]. Ana R. Ribeiro, Arijita Mukherjee, Xuan Hu, Shayan Shafien, Reza Ghodsi , Kun Ele, Sara GeminiPiperni , Canhui Wang, Robert F. Klie, Tolou Shokuhfar, Reza Shahbazian- Yassar, Radovan Borojevic , Luis A. Rocha \& José M. Granjeiro. Bio-camouflage of anatase nanoparticles explored by: In situ highresolution electron microscopy. Nanoscale 9, 10684-10693 (2017).

[20]. AR Ribeiro, S. Gemini-Piperni , R. Travassos, L. Lemgruber , R. C. Silva , A. L. Rossi , M. Farina, K. Anselme, T. Shokuhfar, R. Shahbazian-Yassar, R. Borojevic , L. A. Rocha , J. Werckmann, \& JM Granjeiro . Trojan-Like Internalization of Anatase Titanium Dioxide Nanoparticles by Human Osteoblast Cells. Sci. Rep. 6, 1-11 (2016).

[21]. Cardoso AP, Pinto ML, Pinto AT, Pinto MT, Monteiro C, Oliveira MI, Santos SG, Relvas JB, Seruca R, Mantovani A, Mareel M, Barbosa MA, Oliveira MJ. Matrix metalloproteases as maestros for the dual role of LPS- and IL-10-stimulated macrophages in cancer cell behaviour. BMC Cancer 15, 1-14 (2015). 
[22]. Thery, C.; Amigorena, S.; Raposo, G.; Clayton, A. Isolation and characterization of exosomes from cell culture supernatants and biological fluids. Curr Protoc Cell Biol. 3, 3-22, (2006).

[23]. Couto, Marina; Vasconcelos, Daniela P.; Sousa, Daniela M.; Sousa, Beatriz; ConceiÃ§̃̃fo, Francisco; Neto, Estrela; Lamghari, Meriem; Alves, CecÃlia J. The Mechanisms Underlying the Biological Response to Wear Debris in Periprosthetic Inflammation. Frontiers in Materials, 7, 274 (2020).

[24]. Schoon, Janosch; Hesse, Bernhard; Rakow, Anastasia; Ort, Melanie J.; Lagrange, Adrien; Jacobi, Dorit; Winter, Annika; Huesker, Katrin; Reinke, Simon; Cotte, Marine; Tucoulou, Remi; Marx, Uwe; Perka, Carsten; Duda, Georg N.; Geissler, Sven. Metala-Specific Biomaterial Accumulation in Human Peri-Implant Bone and Bone Marrow. Advanced Science, 20004-12 (2020).

[25]. Shi-Cong Tao \& Shang-Chun Guo. Extracellular vesicles in bone: " dogrobbers " in the "eternal battle field ". Cell Commun. Signal 5, 1-13 (2019).

[26]. Van Niel, G., D'Angelo, G. \& Raposo, G. Shedding light on the cell biology of extracellular vesicles. Nat. Rev. Mol. Cell Biol. 19, 213-228 (2018).

[27]. Masaoutis, Christos; Theocharis, Stamatios. The Role of Exosomes in Bone Remodeling: Implications for Bone Physiology and Disease. Disease Markers, 1-12 (2019).

[28]. Hallab, N. J. \& Jacobs, J. J. Chemokines Associated with Pathologic Responses to Orthopedic Implant Debris. Front Endocrinol 8, 1-10 (2017).

[29]. Stuart B. Goodman, Jiři Gallo, E. F. D. G. \& M. T. Diagnosis and Management of Implant DebrisAssociated Inflammation. Expert Rev. Med. Devices 17, 41-56 (2020).

[30]. Kaufman, A. M., Alabre, C. I., Rubash, H. E. \& Shanbhag, A. S. Human macrophage response to UHMWPE, TiAIV, CoCr, and alumina particles: Analysis of multiple cytokines using protein arrays. $\mathbf{J}$ Biomed Mater Res A 84, 464-474 (2007).

[31].Jukka Pajarinen, Vesa-Petteri Kouri, Eemeli JämsenTian-Fang, Li Jami Mandelin Yrjö T. Konttinen. The response of macrophages to titanium particles is determined by macrophage polarization. Acta Biomaterialia 9, 9229-9240 (2013).

[32]. Wynn, T. A. \& Vannella, K. M. Macrophages in Tissue Repair, Regeneration, and Fibrosis. Immunity 44, 450-462 (2016).

[33]. Vasconcelos, D.M., Ribeiro-da-Silva, M., Mateus, A. et al. Immune response and innervation signatures in aseptic hip implant loosening. J Transl Med 14, 205 (2016).

[34]. Koulouvaris P, Ly K, Ivashkiv LB, Bostrom MP, Nestor BJ, Sculco TP, Purdue PE. Expression profiling reveals alternative macrophage activation and impaired osteogenesis in periprosthetic osteolysis. $J$ Orthop Res 26, (1):106-16 (2008). 
[35]. Kumar S, Meena R, Paulraj R. Role of Macrophage (M1 and M2) in Titanium-Dioxide NanoparticleInduced Oxidative Stress and Inflammatory Response in Rat. Appl Biochem Biotechnol 180, (7):12571275 (2016).

[36]. Dhupal, Madhusmita; Oh, Jae-Min; Tripathy, Dipti Ranjan; Kim, Soo-Ki; Koh, Sang Baek; Park, KyuSang. Immunotoxicity of titanium dioxide nanoparticles via simultaneous induction of apoptosis and multiple toll-like receptors signaling through ROS-dependent SAPK/JNK and p38 MAPK activation. International Journal of Nanomedicine 13, (5), 6735-6750 (2018).

[37]. Théry et al. Minimal information for studies of extracellular vesicles 2018 (MISEV2018): a position statement of the International Society for Extracellular Vesicles and update of the MISEV2014 guidelines. Journal of Extracellular Vesicles 8, (1)-1535750 (2019).

[38]. Mehmet Asim Bilen, Tianhong Pan, Yu-Chen Lee, Song-Chang Lin, Guoyu Yu, Jing Pan, David Hawke, Bih-Fang Pan, Jody Vykoukal, Kavanya Gray, Robert L Satcher, Gary E. Gallick, Li-Yuan Yu-Lee, and SueHwa Lin. Proteomics Profiling of Exosomes from Primary Mouse Osteoblasts under Proliferation versus Mineralization Conditions and Characterization of Their Uptake into Prostate Cancer Cells. J Proteome Res 16, 2709-2728 (2017).

[39]. Dilshan S. Harischandra, Shivani Ghaisas, Dharmin Rokad, Anumantha G. Kanthasamy. Exosomes in Toxicology: Relevance to Chemical Exposure and Pathogenesis of Environmentally Linked Diseases. Toxicol Sci 158, 3-13 (2017).

[40]. Moon PG, Lee JE, Cho YE, Lee SJ, Chae YS, Jung JH, Kim IS, Park HY, Baek MC. Fibronectin on circulating extracellular vesicles as a liquid biopsy to detect breast cancer. Oncotarget 7, 40189-40199 (2016).

[41]. Holliday, L. S., Faria, L. P. De \& Jr, W. J. R. Actin and Actin-Associated Proteins in Extracellular Vesicles Shed by Osteoclasts. Int J Mol Sci 21, 1-158 (2019).

[42]. Sonia A. Melo, Linda B. Luecke, Christoph Kahlert, Agustin F. Fernandez, Seth T. Gammon, Judith Kaye, Valerie S. LeBleu, Elizabeth A. Mittendorf, Juergen Weitz, Nuh Rahbari, Christoph Reissfelder, Christian Pilarsky, Mario F. Fraga, David Piwnica-Worms \& Raghu Kalluri. Glypican-1 Identifies Cancer Exosomes and Detects Early Pancreatic Cancer, Nature 9, 177-82 (2015).

[43]. Juliana P. Vago, Michelle A. Sugimoto, Kátia M. Lima, Graziele L. Negreiros-Lima, Nagyung Baik, Mauro M. Teixeira, Mauro Perretti, Robert J. Parmer, Lindsey A. Miles, and Lirlândia P. Sousa. Plasminogen and the Plasminogen Receptor, Plg-R KT, Regulate Macrophage Phenotypic, and Functional Changes. Front Immunol 10, 1-16 (2019).

[44]. Géza Pap, René Eberhardt, C.Röcken,W.Nebelung, H.W.Neumann, A.Roessner. Expression of Stromelysin and Urokinase Type Plasminogen Activator Protein in Resection Specimens and Biopsies at Different Stages of Osteoarthritis of the Knee. Pathol. Res. Pract 49, 219-226 (2000). 
[45]. Syggelos, S. A., Aletras, A. J., Smirlaki, I. \& Skandalis, S. S. Extracellular Matrix Degradation and Tissue Remodeling in Periprosthetic Loosening and Osteolysis: Focus on Matrix Metalloproteinases, Their Endogenous Tissue Inhibitors, and the Proteasome. Biomed Res Int 1, 230805 (2013).

[46]. Qin Shi, Daniel Lajeunesse, Pascal Reboul, Johanne Martel-Pelletier, Jean-Pierre Pelletier, Faramaze Dehnade and Julio C Fernandes. Metabolic Activity of Osteoblasts from Periprosthetic Trabecular Bone in Failed Total Hip Arthroplasties and Osteoarthritis as Markers of Osteolysis and Loosening. $J$

Rheumatol 29,1437-1445 (2002).

[47]. Nordsletten, L. et al. The plasminogen activation system is upregulated in loosening of total hip prostheses 6470, (2009).

[48]. Daci, E., Udagawa, N., Martin, T. J., Bouillon, R. \& Carmeliet, G. The Role of the Plasminogen System in Bone Resorption In Vitro. J Bone Miner Res 14, 946-52 (1999).

[49]. Jin, T., Tarkowski, A., Carmeliet, P. \& Bokarewa, M. Urokinase, a constitutive component of the inflamed synovial fluid, induces arthritis. Arthritis Res Ther 5, 9-17 (2003).

[50].Emanuela Galliera, Lorenzo Drago, MonicaGioia Marazzi, Carlo Romano, Christian Vassena, MassimilianoM. CorsiRomanelli. Clinica Chimica Acta Soluble urokinase-type plasminogen activator receptor (suPAR) as new biomarker of the prosthetic joint infection: Correlation with in flammatory cytokines. Clin Chim Acta 441, 23-28 (2015).

[51]. RB Raggam, J. Wagner, F. Prüller, A. Grisold, E. Leitner I. Zollner-Schwetz, T. Valentin, R. Krause M. Hoenigl. Soluble urokinase plasminogen activator receptor predicts mortality in patients with systemic inflammatory response syndrome. J. Intern. Med 276, 651-658 (2014).

[52]. Rosso, M. Del, Fibbi, G., Pucci, M., Margheri, F. \& Serratì, S. The plasminogen activation system in inflammation. Front Biosci 1, 4667-4686 (2008).

\section{Figures}



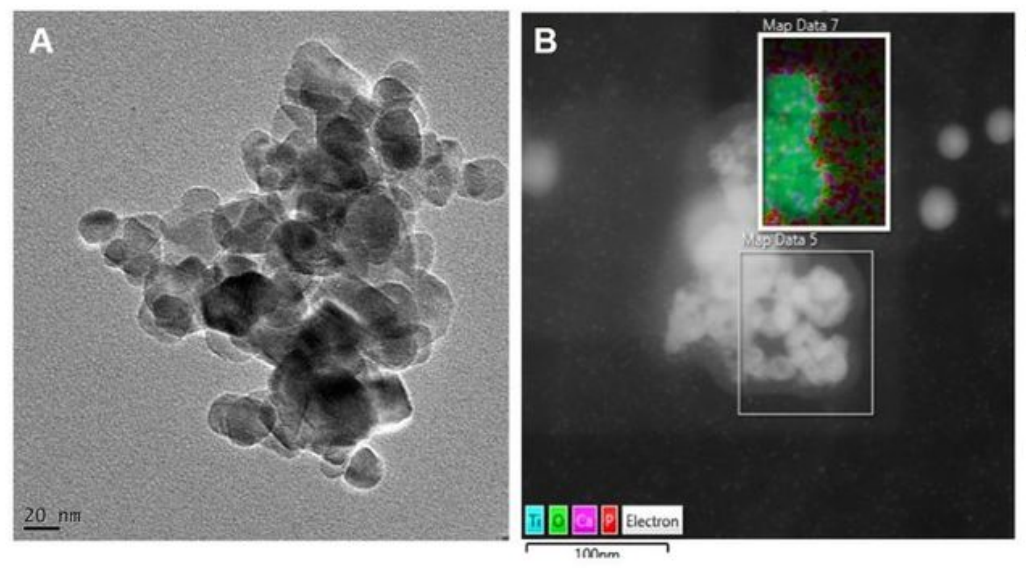

\begin{tabular}{|c|c|}
\hline \multicolumn{2}{|c|}{ Titanium dioxide nanoparticles $\left(\mathrm{TiO}_{2} \mathrm{NPs}\right)$} \\
\hline crystal phase & Anatase \\
\hline \multicolumn{2}{|c|}{ Size } \\
\hline Size Sigma & $<25 \mathrm{~nm}$ \\
\hline DLS Size in water & $113 \pm 1 \mathrm{~nm}$ \\
\hline DLS Size in medium culture & $143 \pm 5 \mathrm{~nm}$ \\
\hline TEM Size in water & $118 \pm 3 \mathrm{~nm}$ \\
\hline TEM Size medium culture & $149 \pm 6 \mathrm{~nm}$ \\
\hline \multicolumn{2}{|c|}{ Surface area } \\
\hline Brunaur-Emmett-Teller (BET) & $61.1 \pm 0,2 \mathrm{~m}^{2} / \mathrm{g}$ \\
\hline \multicolumn{2}{|c|}{ Potencial zeta } \\
\hline Potential Zeta in water & $-22,2 \mathrm{mV}$ \\
\hline Potential Zeta in medium culture & $-6,42 m V$ \\
\hline \multicolumn{2}{|c|}{ Morphology } \\
\hline & \\
\hline
\end{tabular}

D In vitro studies

Ultracentrifugation and Density Gradient

In vitro studies

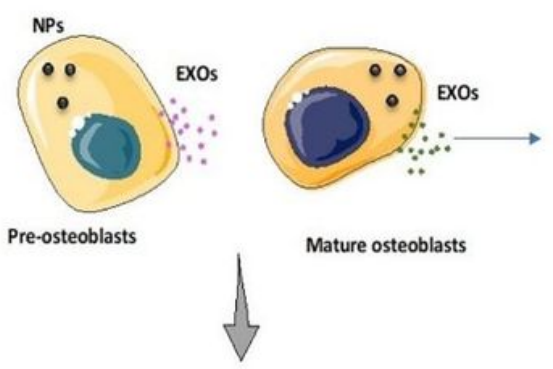

Effect of NPs on cells
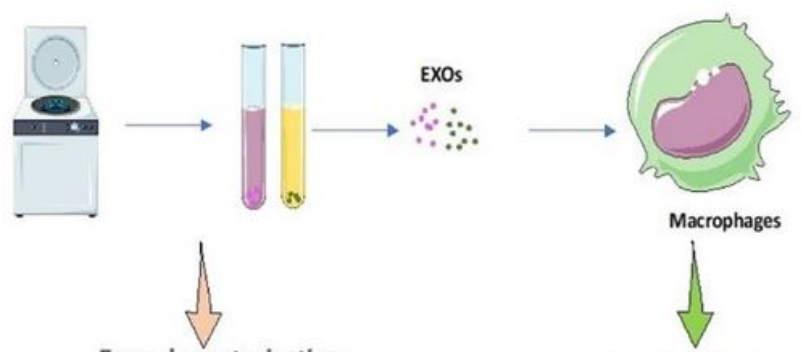

$$
\begin{aligned}
& \text { Viability } \\
& \text { Proliferation } \\
& \text { NPs internalization }
\end{aligned}
$$

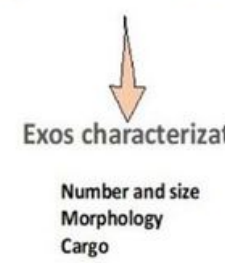

Functional tests

Viability

Cytokine production

Morphology

Surface Markers

\section{Figure 1}

Characterization of TiO2 NPs: (A) Transmission electron micrograph (TEM) showing the primary size of TiO2 NPs and its agglomeration in water after 24 hours of dispersion (scale bar: $20 \mathrm{~nm}$ ). (B) TEM and STEM-EDS element map showing TiO2 NPs in medium culture with calcium (Ca) and phosphorus (P) adsorption (scale bar: $100 \mathrm{~nm}$ ). (C) Table with the main physical and chemical characteristics of the TiO2 NPs in water and medium culture. (D) Schematic representation of the workflow: exposure of TiO2 NPs to pre (MG63) and mature osteoblasts (SAOS-2), isolation of Exos and functional tests on macrophages. 
A

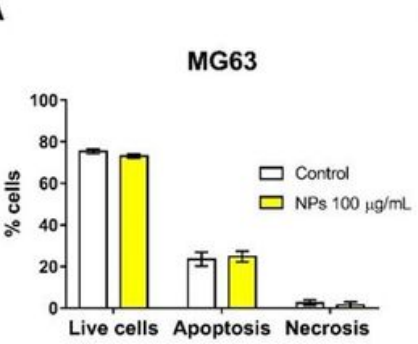

C

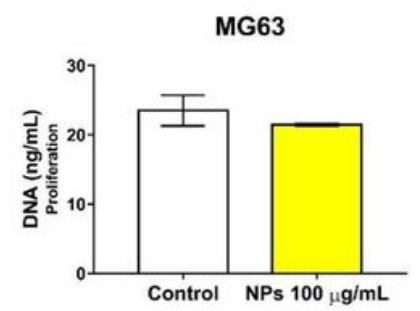

B

SAOS-2

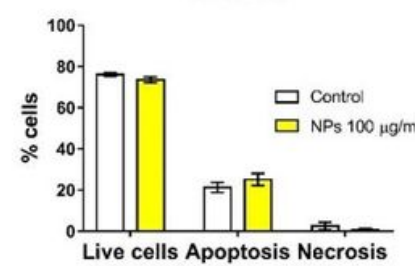

D

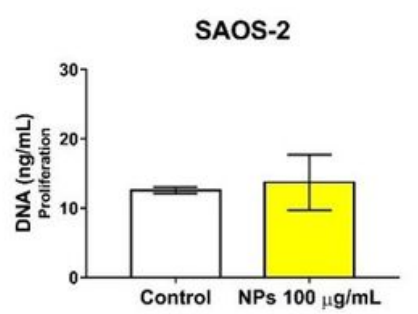

E

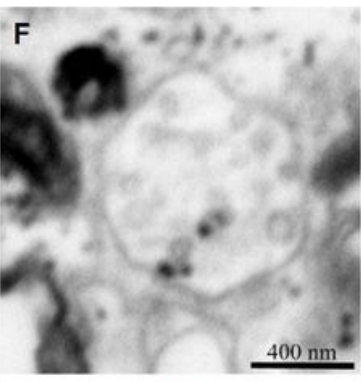

G

\section{Figure 2}

The effect of TiO2 NPs on osteoblasts behavior: (A) Effect of TiO2 NPs on MG63 (pre-osteoblastic cell line) and (B) SAOS-2 (mature osteoblasts) cell viability/apoptosis/necrosis (flow cytometry analysis) and proliferation ( $C$ and $D)$. ( $E$ ) Transmission electron representative micrographs of TiO2 NPs internalization on MG63 revealing internalization of NPs in (F) multivesicular bodies (MVBs), (G) where NPs enter in direct contact with Exos; a 3D reconstruction of the vesicles obtained by focus ion beam. (Scale bar: 1 $\mu \mathrm{m}, 400 \mathrm{~nm}$ and $200 \mathrm{~nm}$ ). The results represent the mean \pm standard deviation of an independent experimental performed in triplicate. 
A

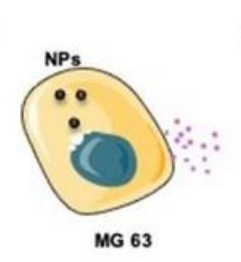

B

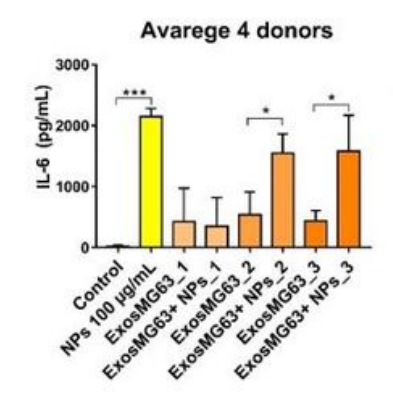

D

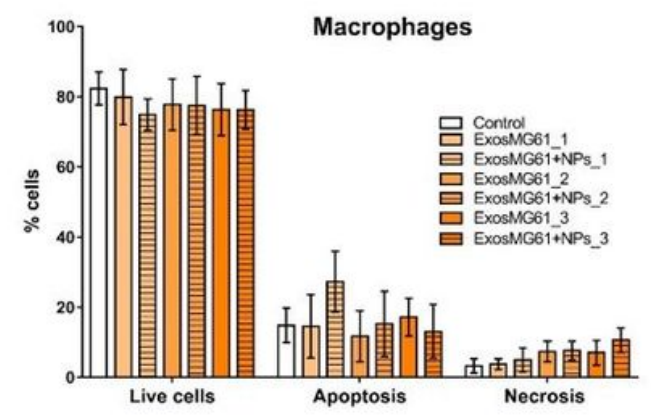

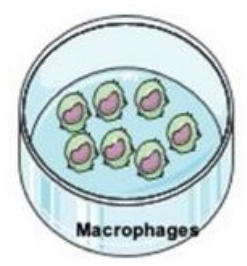
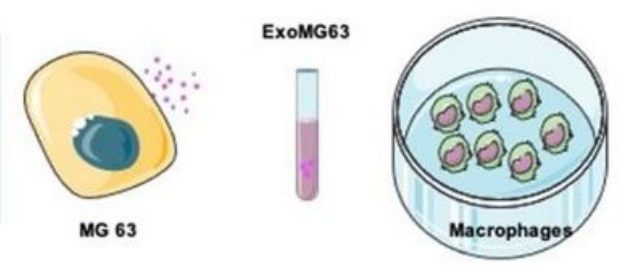

C
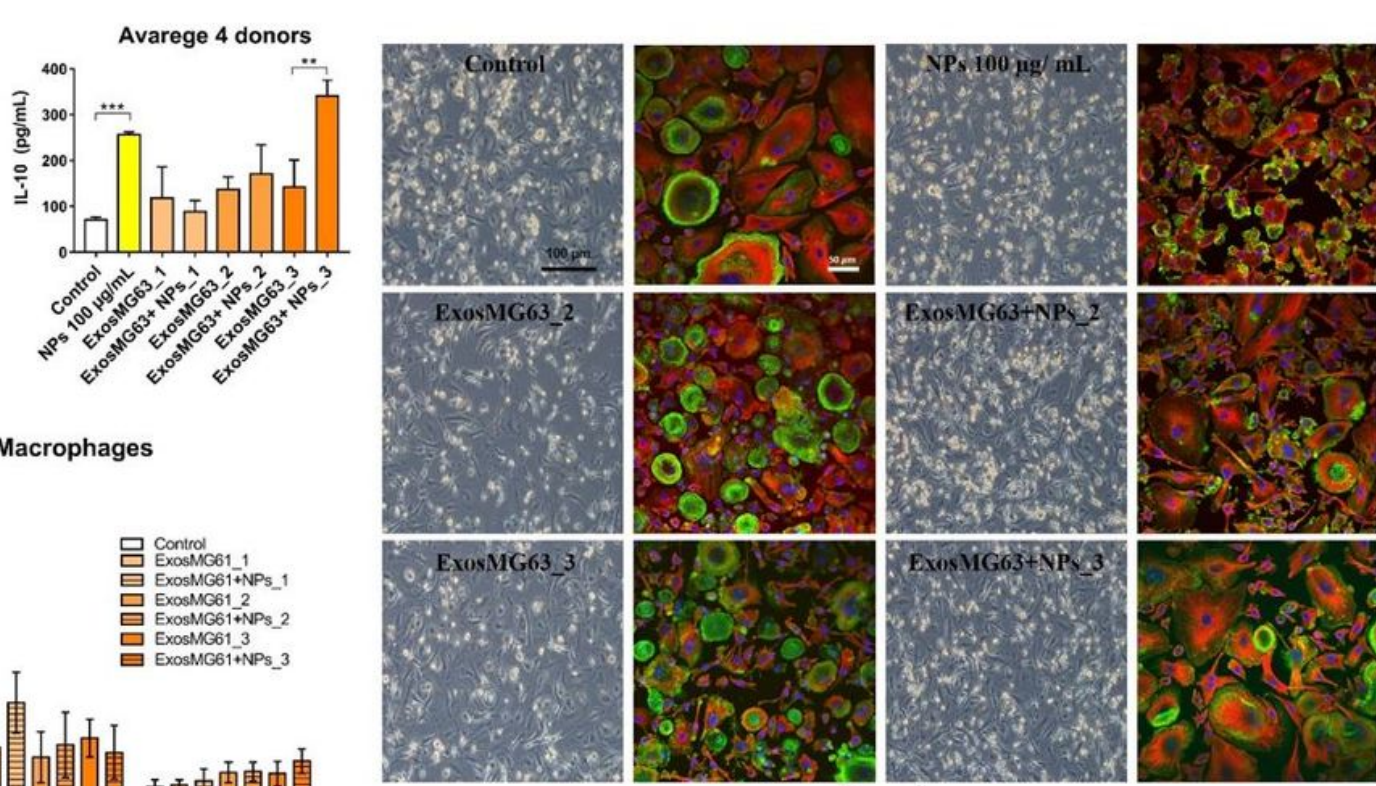

Donor 2

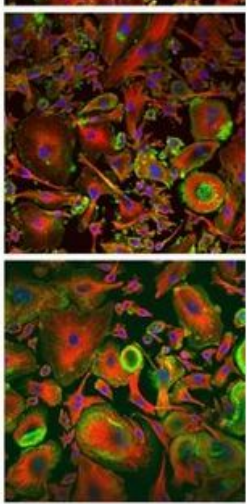

\section{Figure 3}

Exosomes derived from pre-osteoblasts (MG63) induce the secretion of pro and anti-inflammatory cytokines by macrophages: (A) Schematic representation of the treatment of macrophages with Exos derived from MG63 after exposure to different concentrations of TiO2 NPs (ExoMG63+NPs_1: 5) and without exposure to NPs (ExoMG63_1:5). Three concentrations of Exos derived from MG63 not exposed (ExoMG63_1:3) and exposed to TiO2 NPs (ExoMG63+NPs_1:3) were used to stimulate human macrophages: 4 x 103 Exos/mL (ExosMG63_1 and ExoMG63+NPs_1), 4 x 105 Exos/mL (ExosMG63_2 and ExoMG63+NPs_2) and 4 x 107 Exos/mL (ExosMG63_3 and ExoMG63+NPs_3). As control of all the experiments, we analyzed macrophages not exposed (control) and exposed to $100 \mu \mathrm{g} / \mathrm{mL}$ of TiO2 NPs during 72h (NPs $100 \mu \mathrm{g} / \mathrm{mL}$ ). (B) Human macrophages from 4 healthy donors were exposed to different concentrations of MG63 derived Exos upon which pro- (IL-6) and anti-inflammatory (IL-10) cytokines were determined by ELISA in the supernatants. Data are presented as average obtained in the 4 donors. (C) Representative phase-contrast (scale bar: $100 \mu \mathrm{m}$ ) and confocal microscopy images (scale bar: $50 \mu \mathrm{m}$ ) of macrophages stimulated or not (control) with Exos derived from MG63. Macrophage's actin cytoskeleton is illustrated in green, tubulin in red, while nuclei are counterstained with DAPI (in blue). (D) Macrophages viability upon MG63 derived Exos treatment was determined by flow cytometry. Statistical analyzes were performed by ANOVA, followed by multiple comparisons, using the GraphPad Prism 
software. The results represent the mean \pm standard deviation of at least three independent experiments performed in triplicate. *statistical differences between the groups marked.

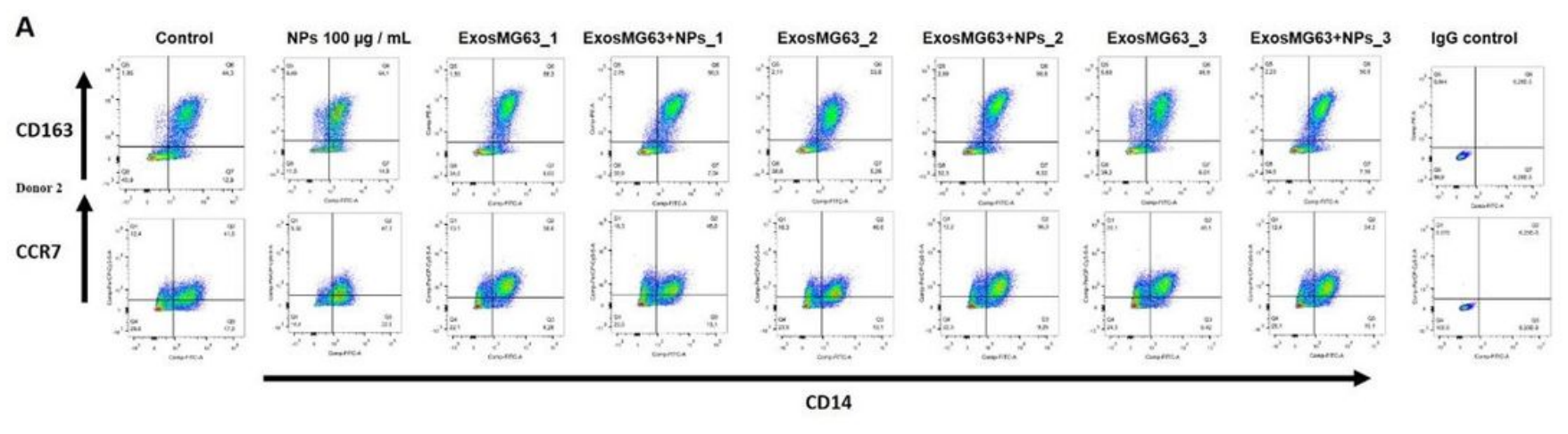

B

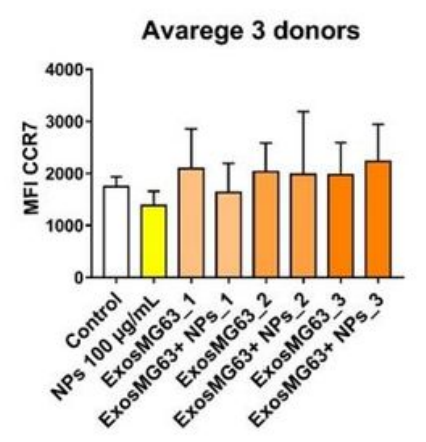

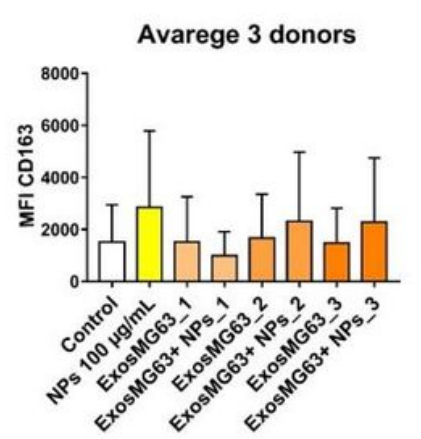

C

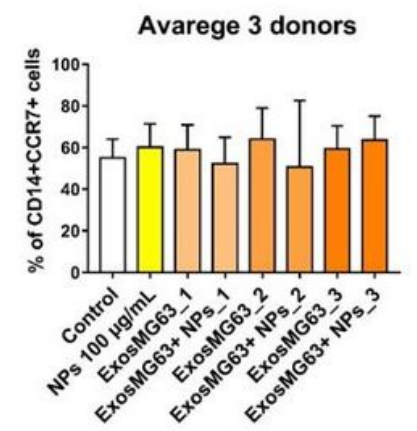

\section{Figure 4}

Osteoblasts-derived exosomes induce a mixed macrophage polarization: Macrophages were differentiated for 10 days and then treated for $72 \mathrm{~h}$ with Exos isolated from MG63 cells previously incubated or not with TiO2 NPs. By flow cytometry, CD14 was used as a macrophage lineage marker and CD163 and CCR7 were used as M2 and M1-associated markers, respectively. Representative dot plots illustrate the gates used to identify CD14+CD163+ cells and CD14+ CCR7+ cells (FlowJo software). (A) Numbers indicate the percentage of the double positive populations. (B) The mean fluorescence intensity (MFI) of CCR7 and CD163 was determined by subtraction of the fluorescence intensity of the respective isotype control. (C) The percentage of double positive cells (CD14+CCR7+ and CD14+CD163+) was also determined. Statistical analyzes were performed by ANOVA, followed by multiple comparisons, using GraphPad software. The results represent the mean \pm standard deviation of values obtained with macrophages from at least 3 different donors. 
A

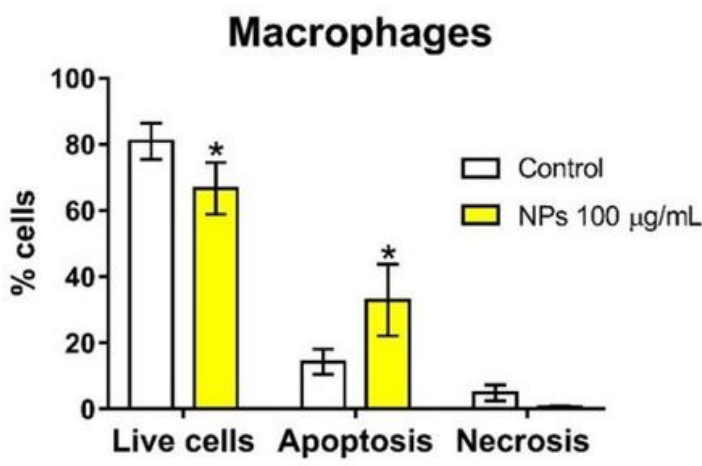

B

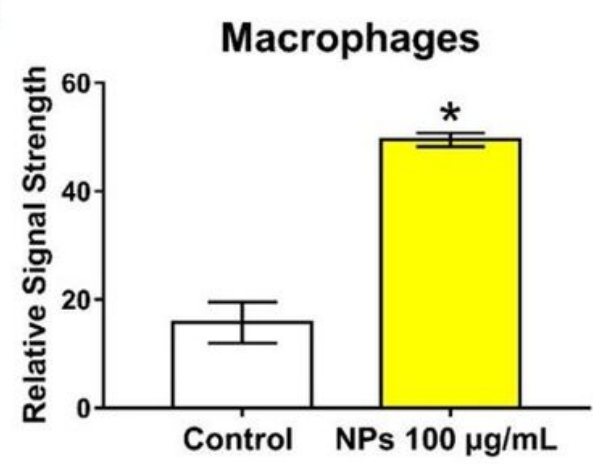

C

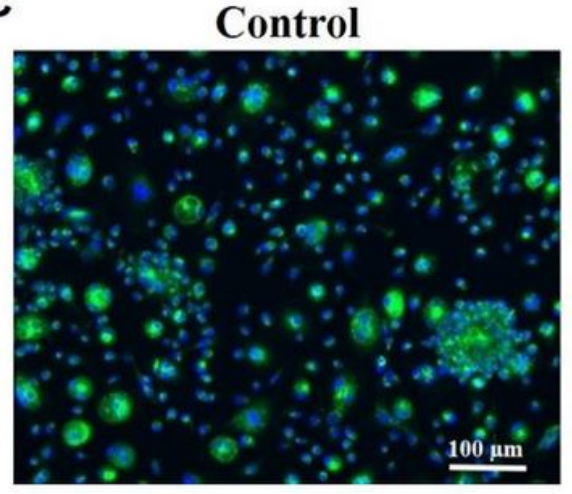

NPs $100 \mu \mathrm{g} / \mathrm{mL}$

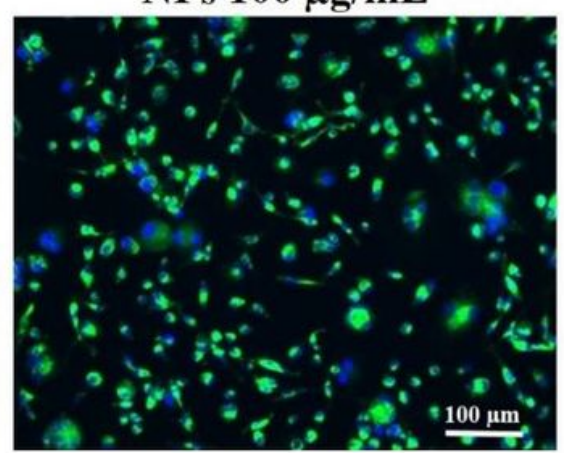

D

NPs $100 \mu \mathrm{g} / \mathrm{mL}$

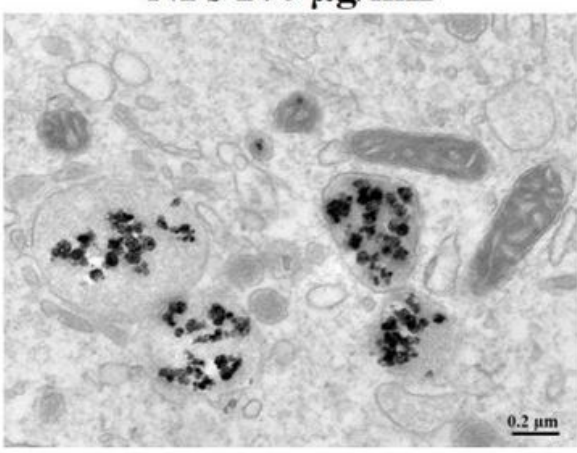

Figure 5

The direct effect of TiO2 NPs on macrophage behavior: (A) Effect of TiO2 NPs on Human macrophage cell viability/apoptosis/necrosis (flow cytometry analysis) upon NPs stimulation. (B) Quantification of reactive oxygen species (ROS) production after treatment with TiO2 NPs. (C) Fluorescent micrographs showing the lysosomes activity (green) and nucleus (blue) (scale bar: $100 \mu \mathrm{m}$ ). (D) Transmission electron representative micrographs of TiO2 NPs internalization in Human macrophage revealing internalization of NPs (scale bar: $0.2 \mu \mathrm{m}$ ). The results represent the mean \pm standard deviation of an independent experimental performed in triplicate. *statistical differences between the groups marked. 

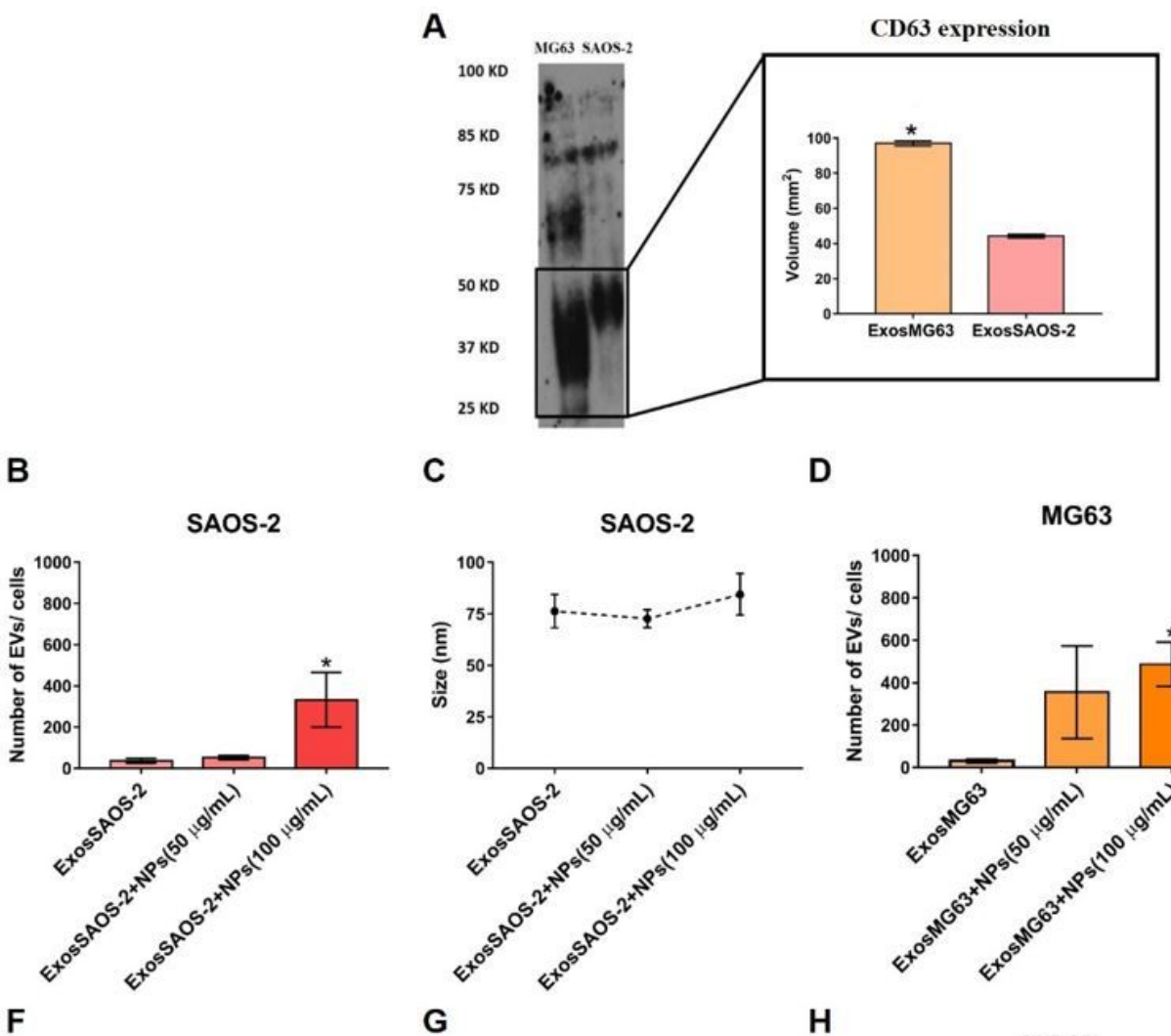

C

D
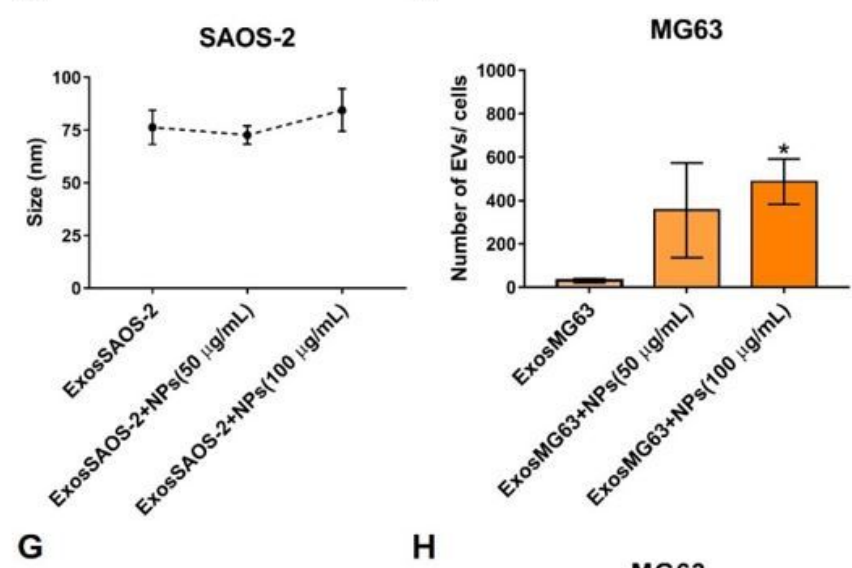

E
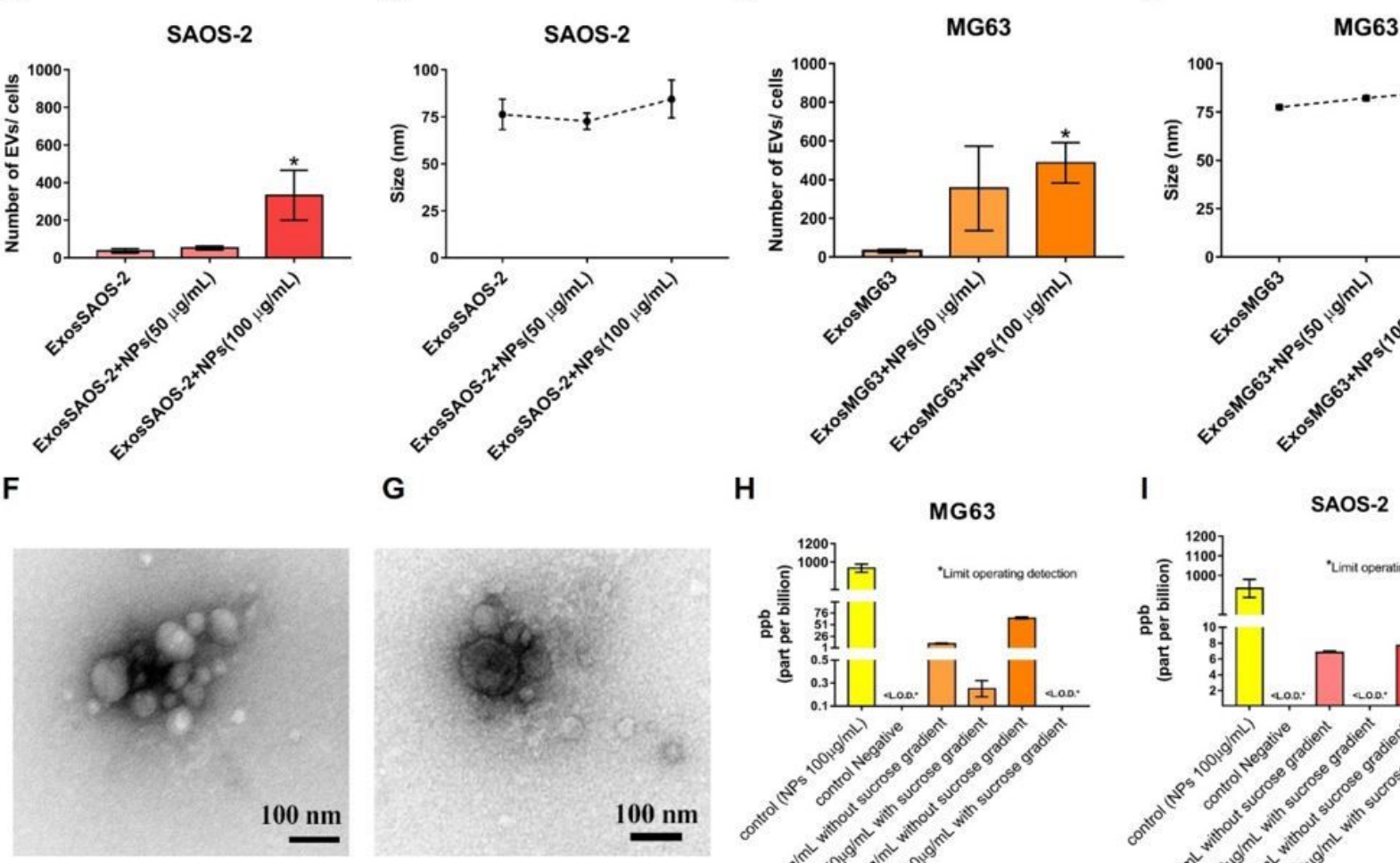

G

H

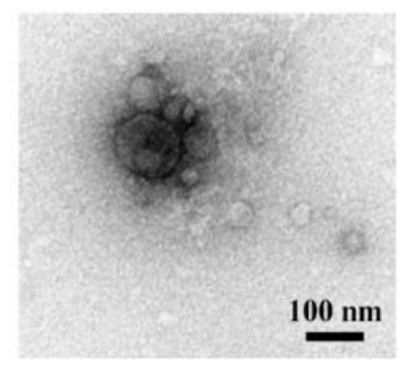

H

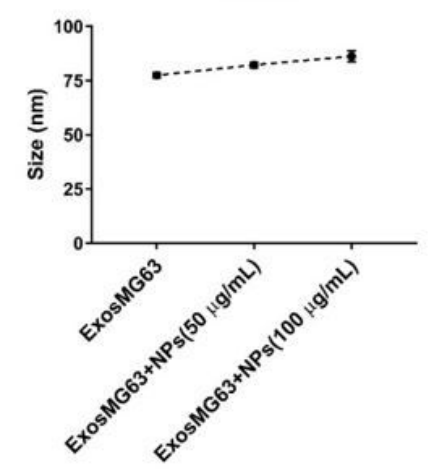

I

MG63
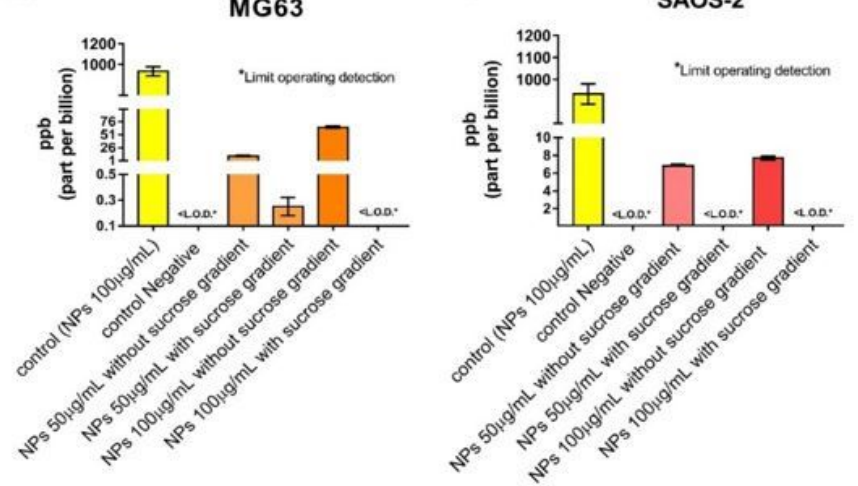

\section{Figure 6}

TiO2 NPs stimulated an increase in exosomes secretion: Exos isolation from MG63 (ExoMG63+NPs) and SAOS-2 (ExosSAOS-2+NPs) after exposure to different concentrations of TiO2 NPs, where ultracentrifugation and a sucrose gradient was employed. As a control, the same protocol of isolation was employed without NPs exposure (ExosMG63 and ExosSAOS-2). (A) Identification and quantification of CD63 marker by western blot and densitometry analysis confirming the isolation of Exos derived from MG63 and SAOS-2. (B) Number and (C) the size of Exos derived from MG63 and SAOS-2 (D and E) exposed or not (control) to TiO2 NPs obtained by nanoparticle size analysis. (F and G) Representative micrographs obtained by Transmission Electron Microscopy revealing the morphology of Exos derived from MG63 and SAOS-2 pre-incubated with TiO2 NPs. Quantification of titanium traces by ICP-MS in Exos derived from $(\mathrm{H}) \mathrm{MG63}$ and (I) SAOS-2 (pre-incubated or not with TiO2 NPs) after sucrose gradient purification. * ppb (part per billion). Statistical analyzes were performed by ANOVA, followed by multiple 
comparisons. The results represent the mean \pm standard deviation of at least 5 independent experiments carried out in triplicate. *Statistical differences between the groups marked.

A

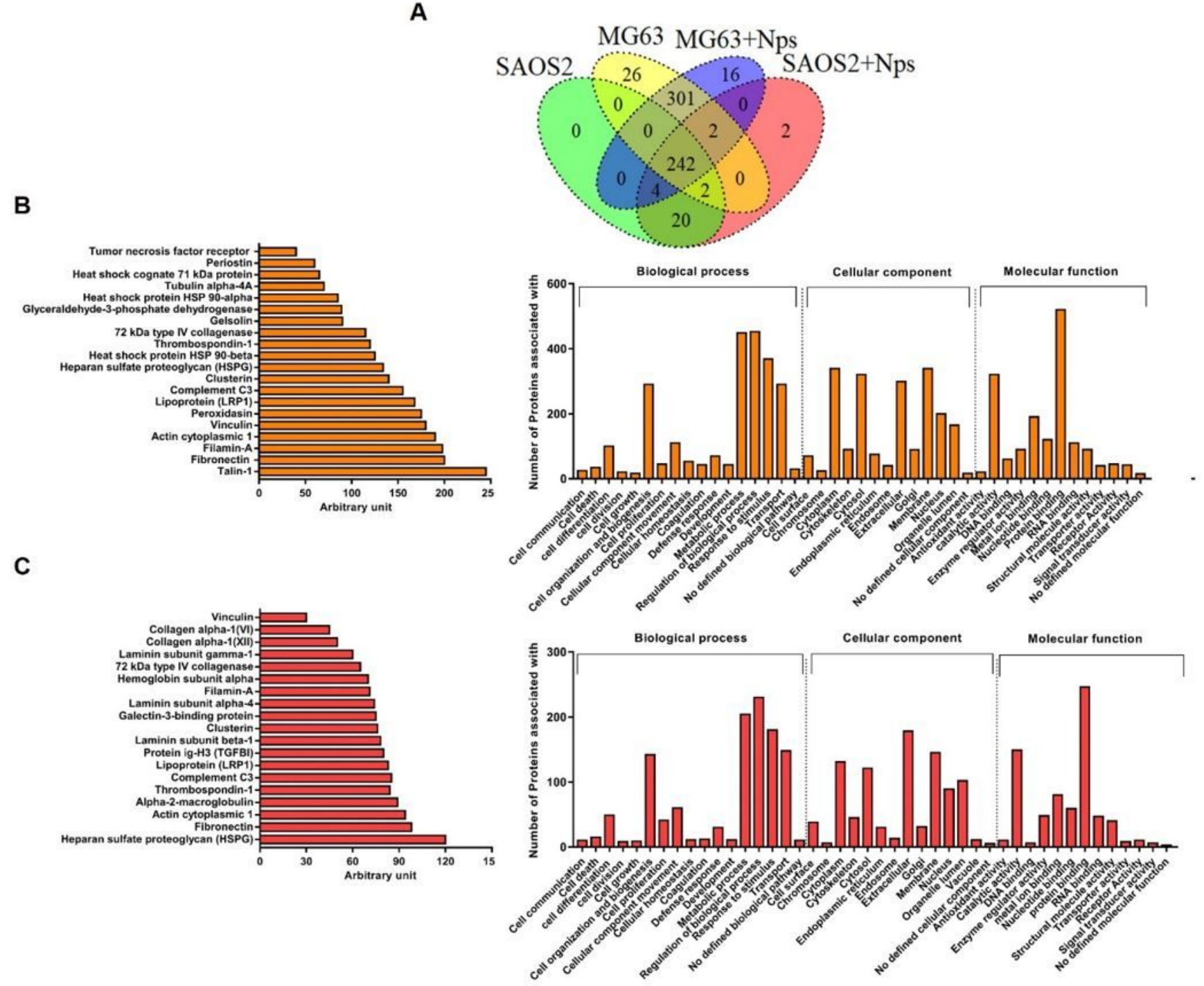

Figure 7

TiO2 nanoparticles alter MG63 and SAOS-2 derived exosomes cargo: (A)Veen diagram of identified proteins from three replicate samples of Exos derived from MG63 and SAOS-2 exposed or not to TiO2 NPs by LC-MS/MS. Top twenty most expressed exosomal proteins derived from (B) ExosMG63+NPs and (C) ExosSAOS-2+NPs with the gene ontology classification on the basis of their involvement on biological processes, cellular components and molecular function. The samples were analyzed in the Uniprot database for taxonomic selection towards Homo sapiens. 
A
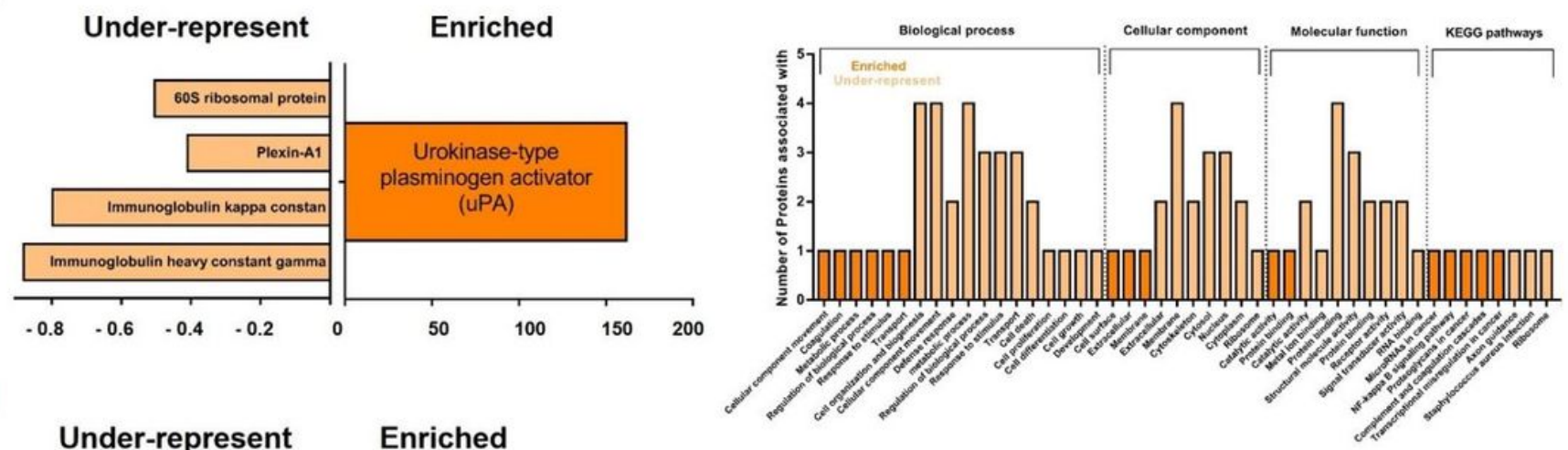

B
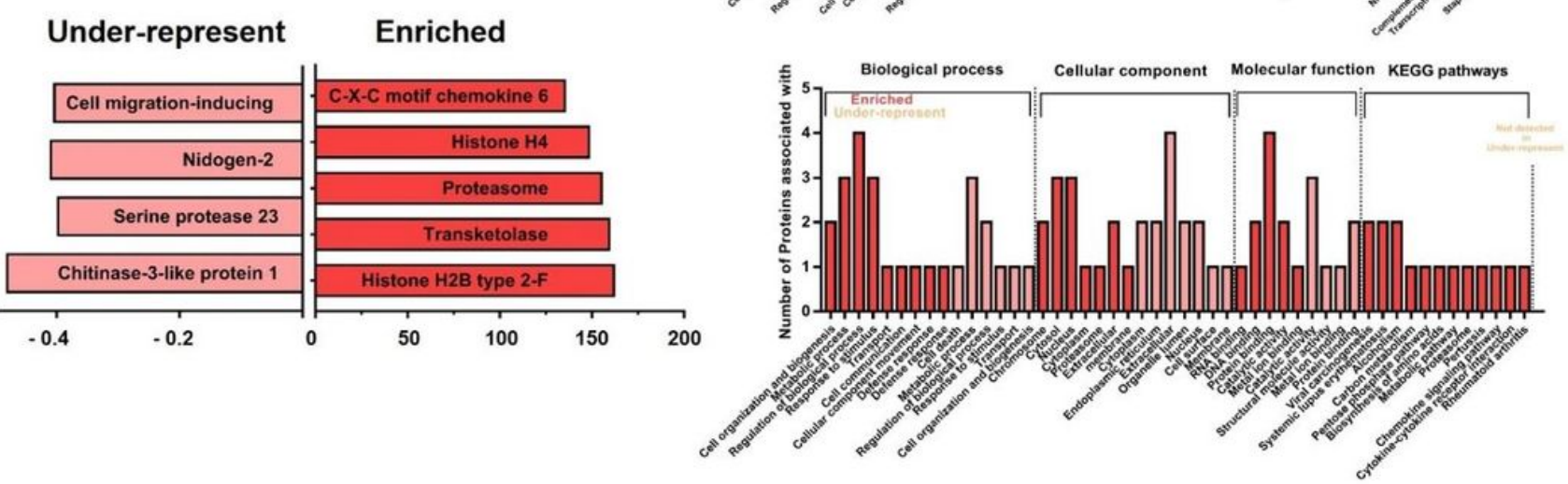

\section{Figure 8}

TiO2 nanoparticles alter MG63 and SAOS-2 derived exosomes cargo: Identified clusters that are unique and that are sometimes under-represented and/or enriched in Exos derived from (A) MG63 exposed to NPs (ExosMG63+NPs) and (B) SAOS-2 (ExosSAOS-2+NPs). The samples were analyzed in the Uniprot database for taxonomic selection towards Homo sapiens. Considering the control ( $p$ 0.01), a list of proteins that increased and decreased after the treatment of TiO2 NPs was identified. 


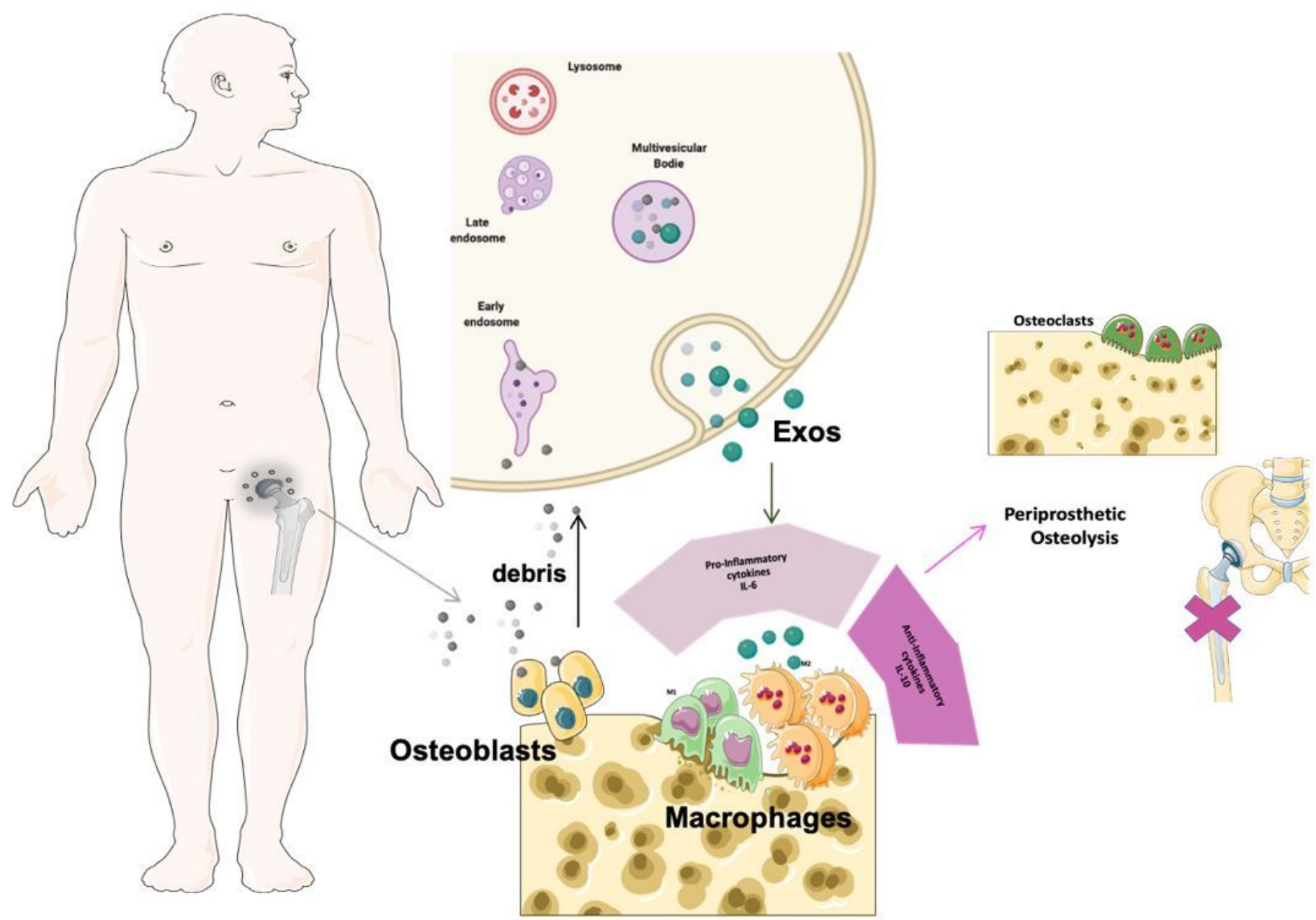

Figure 9

Schematic model of the use of exosomes as new biomarkers of joint prosthesis failure: Titanium implantable systems due to wear processes in human body start to release nano wear debris at the interface implant bone. These TiO2 NPs interact with osteoblasts with different degrees of maturation that are present at the bone-implant interface. These TiO2 NPs are internalized by bone cells and localized directly in MVBs altering Exos biogenesis and cargo. Pre-osteoblastic derived Exos enriched with UPA stimulate macrophages towards an inflammatory pathway that was already reported in periprosthetic osteolysis. The fact that Exos are considered as shuttle vesicles involved in cell communication possibly can contribute to a systemic toxicity.

\section{Supplementary Files}

This is a list of supplementary files associated with this preprint. Click to download.

- sfig1.jpg

- sfig2.jpg

- sfig3.jpg 
- sfig4.jpg

- sfig5.jpg

Page $32 / 32$ 